\title{
The formation of galaxy bulges: Spectrophotometric constraints $^{\star, \star \star}$
}

\author{
Ph. Prugniel ${ }^{1}$, G. Maubon ${ }^{2}$, and F. Simien ${ }^{1}$ \\ 1 CRAL-Observatoire de Lyon, CNRS: UMR 142, 69561 St-Genis-Laval Cedex, France \\ 2 OMP, CNRS: UMR 5572, 14 avenue Edouard Belin, 31400 Toulouse, France
}

Received 23 May 2000 / Accepted 24 October 2000

\begin{abstract}
We have measured $\mathrm{Mg}_{2}$, Fe 5270 and Fe 5335 spectrophotometric indices (LICK system) in the bulge of 89 galaxies, mostly spirals from the Héraudeau (1996) sample. The indices are reduced to a null velocity dispersion and normalized to an aperture of $0.2 \mathrm{~h}^{-1} \mathrm{kpc}$. The mean errors are $0.009 \mathrm{mag}$ on $\mathrm{Mg}_{2}$, and $0.3 \AA$ on the iron indices. These measurements almost double the amount of similar data already available on spiral galaxies. Our data confirm the existence of the relation between $\mathrm{Mg}_{2}$ and $\sigma_{0}$, the central stellar velocity dispersion; we find an even tighter relation between $\mathrm{Mg}_{2}$ and $V_{\text {rot }}^{\mathrm{m}}$, the maximum rotational velocity of the galaxy, deduced from HI observations. For the most massive bulges, these correlations may be interpreted as a mass-metallicity relation. However, the presence of young stellar populations, traced by the detection of [OIII] $\lambda 5007 \AA$ emission, provides clear evidence that age effects do play a role. Since the contribution of the young population is anti-correlated to the mass of the galaxy, it continues the $\mathrm{Mg}_{2}$ vs. $\sigma_{0}$ relation toward the low- $\sigma_{0}$ region and globally increases its slope. We also present evidence for a new positive correlation between Fe indices and $\sigma_{0}$, and for a significant correlation between the line-strength indices and the total or disk luminosity. We propose to model the whole sequence of bulges within the following framework: bulges are composed of a primary population formed prior to the disk, during the initial collapse, and of a secondary population formed during its evolution. The whole family of bulges can be classified into three classes: (A) the bulges dominated by young populations are generally small, have ionized gas, low velocity dispersion and low line strengths; (B) the bulges dominated by the primary population lie along the mass-metallicity sequence defined for elliptical galaxies; and (C) the bulges where the secondary population is significant are less Mg-over-abundant than (B)-type bulges and deviate from the $\mathrm{Mg}_{2} \quad \mathrm{vs}$. $\sigma_{0}$ relation of elliptical galaxies.
\end{abstract}

Key words. galaxies: general - galaxies: spiral - galaxies: fundamental parameters - galaxies: stellar content galaxies: evolution

\section{Introduction}

Studying the stellar populations in bulges addresses the key question for understanding the formation processes of galaxies. Do the bulges form by dissipative collapse, as suggested for ellipticals? What is the contribution of the secular evolution driven by a star formation triggered by bars? Goudfrooij et al. (1999) and Wyse et al. (1997) have reviewed these questions.

There are presently two main scenarios to explain the origin of bulges (Bouwens et al. 1999). The first is

Send offprint requests to: Ph. Prugniel,

e-mail: prugniel@obs .univ-lyon1.fr

* Based on observations collected at the Observatoire de Haute-Provence.

** Table 3 is presented in electronic form only at the CDS. Tables 1 and 2 are also available form at the CDS, Strasbourg, via anonymous ftp to cdsarc.u-strasbg.fr (130.79.128.5) or via http://cdsweb.u-strasbg.fr/cgi-bin/qcat?J/A+A/366/68
"Monolithic Collapse", where bulges form from "pregalactic" gas. The collapse of this gas creates stars and the disk comes after, from an infall of halo gas. Observational evidence (Carollo et al. 1993) and models (Carlberg 1984) suggest that early-type galaxies also formed by dissipative collapse. The similarity (or continuity) between elliptical galaxies and bulges of S0 and spiral galaxies is a strong argument in favor of a common origin for all the spheroidal stellar systems. This connection is in particular apparent in the surface-brightness vs. luminosity diagram (Kormendy 1985) and in the central velocity dispersion vs. magnesium index, $\sigma_{0}$ vs. $\mathrm{Mg}_{2}$, relation (Bender et al. 1993; Fisher et al. 1996; Jablonka et al. 1996, hereafter JMA96; Idiart et al. 1996b, hereafter IPC96). Bulges, as well as elliptical galaxies, appear to be over-abundant in $\mathrm{Mg}$ with respect to $\mathrm{Fe}$ - or, adopting a recent view, underabundant in $\mathrm{Fe}$ with respect to the so-called " $\alpha$ " or " $\mathrm{E}$ " elements (Worthey 1998; Trager et al. 2000a); this fact 
is considered as convincing evidence that star formation occurred within a time shorter than the onset of TypeIa supernovae (i.e. shorter than 1 or 2 Gyr). Finally, a growing bulk of evidence that large bulges, including the Galactic and M 31 bulges, are old and have a narrow dispersion in their age (Peletier et al. 1999; Abraham et al. 1999) supports the monolithic collapse scenario.

The second scenario is the "Secular Evolution" model, where the bulge is created from disk material through the action of a stellar bar, clump migration, interactions between galaxies or merging. Observations as well as numerical experiments clearly reveal the link between the bar phenomenon and star formation (e.g. Martinet \& Friedli 1997; Aguerri 1999). Friedli et al. (1995, 1993) showed, with numerical simulations, that a stellar bar is able to drive a large amount of gas to the center and trigger intense star formation. This bar can be dissolved by sufficient central mass concentration (Hasan et al. 1990; Norman et al. 1996) and leave a stellar bulge (or increase the mass of a pre-existing bulge). In a different approach, Noguchi (1999) proposed that the bulge is assembled from massive clumps formed within the disk. Mergers, and probably interactions, are also possibly consistent with the observed correlations between bulge and disk characteristics (Kauffmann 1996).

Several recent observational results (Balcells \& Peletier 1994; see Wyse 1999 for a review) lead to an intermediate view, where large bulges are formed by dissipative collapse with the disk forming later, and small bulges are significantly affected by the evolution.

Although the stars making up the bulge may have formed in the disk and have migrated to the bulge later due to dynamical instabilities, the history of this stellar population clearly conceals important clues about the formation of the bulges.

In order to contribute new arguments to the debate, we report in the $\mathrm{Mg}_{2}$, Fe 5270 and Fe 5335 spectrophotometric indices for 89 galaxies mostly belonging to the Héraudeau et al. sample (Héraudeau 1996; Héraudeau \& Simien 1998; Héraudeau et al. 1999: hereafter collectively referred to as H96). The spectra of these galaxies are available in the library of medium-resolution spectra published in a series of papers (Prugniel \& Simien 1994; Simien \& Prugniel 1997a, 1997b, 1997c), Héraudeau \& Simien (1998, hereafter H1) and Héraudeau et al. (1999, hereafter H2). The present sample will more than double the number of bulges with measured line-strength indices; the previous samples of Fisher et al. (1996), JMA96 and IPC96 count respectively 14, 20 and 45 objects. Older studies concern early-type galaxies (e.g. Bender et al. 1993, 19 bulges).

The sample and data are presented in Sect. 2, the measurement method in Sect. 3, the standardization and final measurements and external comparison in Sect. 4; Sect. 5 uses these new data to revisit the discussion on the formation of bulges.

\section{Sample and observations}

\subsection{Sample}

The sample (Table 1) was originally selected for kinematical and morphological studies. It is composed of 89 galaxies of types Sa to Sc, barred and unbarred (as far as the RC3 classification may be trusted to assert the presence of a bar), and a few S0 galaxies. They are mostly Virgo members and field objects. This sample includes nearby $\left(c z<4000 \mathrm{~km} \mathrm{~s}^{-1}\right.$, with a mean of $\left.\approx 2000 \mathrm{~km} \mathrm{~s}^{-1}\right)$, bright $\left(-23 \leq M_{\mathrm{B}} \leq-18\right)$ galaxies but does not claim completeness in this volume. Kinematical data extracted from these spectra were published in $\mathrm{H} 1$ and $\mathrm{H} 2$, and are available in the Hypercat database ${ }^{1}$. The central velocity dispersion is in the range $50 \leq \sigma_{0} \leq 220 \mathrm{~km} \mathrm{~s}^{-1}$. A well-known Seyfert galaxy, NGC 3227, was also observed; it is reported in the tables, but it will be excluded from the discussion.

\subsection{Data}

The H1 and $\mathrm{H} 2$ observations were obtained with the CARELEC $^{2}$ long-slit spectrograph, mounted on the 1.93$\mathrm{m}$ telescope at the Observatoire de Haute-Provence. The detector was a Tektronix CCD with $512 \times 512$ pixels of $27 \mu \mathrm{m}$. The slit dimensions, projected onto the sky, were $2.2^{\prime \prime}$ in width and $5.5^{\prime}$ in length. The spatial scale was $1.1^{\prime \prime} \mathrm{px}^{-1}$. The $600 \mathrm{gr} \mathrm{mm}^{-1}$ grating provided a dispersion of $66 \AA \mathrm{mm}^{-1}$, equivalent to about $1.77 \AA \mathrm{px}^{-1}$. The spectra are centered on the $\operatorname{Mg} b \lambda 5175 \AA$ triplet, and the spectral coverage is about $900 \AA$. The spectroscopic FWHM resolution is $3.2 \AA$ (or $1.8 \mathrm{px}$ ).

The datasets used for this paper are listed in Table 2 and the spectra are available in the Hypercat FITS archive (hereafter HFA). A total of 155 spectra were secured by Philippe Héraudeau in the period from January 1993 to January 1995, during five observation runs (this includes the two spectra of NGC 3227).

We did not use the two observing runs from $\mathrm{H} 1$ taken with the $1200 \mathrm{gr} \mathrm{mm}^{-1}$ grating $\left(33 \AA \mathrm{mm}^{-1}\right)$ because the spectral interval is not always sufficient to make the $\mathrm{Mg}_{2}$ measurements. Typically, two 45-min exposures were obtained for each galaxy. Several template stars were observed during each run.

\section{Measurements on individual spectra}

\subsection{Data reduction and extraction of spectra}

The spectra archived in HFA contain all the information (in the form of dedicated FITS keywords) needed to enter automatic pipelines producing fully reduced extracted (i.e. 1D) spectra. Each archived file contains pointers to locate bias, flat-field, wavelength- and flux-calibration relations to be used. The center of the object was determined on each raw spectrum by fitting a Gaussian to the mean

\footnotetext{
1 http://www-obs.univ-lyon1.fr/hypercat/

2 http://www.obs-hp.fr
} 
Table 1. Catalog elements and final measurements for our 89 galaxies, and four additional objects

\begin{tabular}{|c|c|c|c|c|c|c|c|c|c|c|c|c|}
\hline Object & Type & $V_{\text {dist }}$ & $\overline{V_{\text {rot }}^{\mathrm{m}}}$ & $\sigma_{0}$ & $R_{6}^{\prime}$ & $N_{\text {obs }}$ & $\mathrm{Mg}_{2}$ & \pm & OIII & \pm & $<\mathrm{Fe}>$ & \pm \\
\hline$(1)$ & $(2)$ & $(3)$ & $(4)$ & (5) & $(6)$ & (7) & $(8)$ & (9) & $(10)$ & (11) & $(12)$ & (13) \\
\hline IC 0356 & $\mathrm{Sb}$ & 1565. & 337 & 141 & 0.481 & 1 & 0.2568 & 0.0063 & 0.42 & 0.12 & 2.314 & 0.227 \\
\hline IC 0750 & Sab & 1364. & 170 & 119 & & 2 & 0.1453 & 0.0101 & 2.51 & 0.20 & 1.874 & 0.229 \\
\hline IC 2166 & $\mathrm{SBc}$ & 3243 . & 198 & & 0.196 & 1 & 0.1817 & 0.0270 & 0.89 & 0.25 & 2.157 & 1.178 \\
\hline IC 2327 & $\mathrm{Sa}$ & 3199. & 134 & 115 & & 1 & 0.1015 & 0.0123 & 3.15 & 0.28 & 1.612 & 0.559 \\
\hline NGC 0063 & $\mathrm{~S} ?$ & 1383. & 138 & & 0.034 & 1 & 0.0607 & 0.0101 & 3.83 & 0.19 & 1.590 & 0.444 \\
\hline NGC 0169 & $\mathrm{Sb}$ & 4436. & 245 & 178 & & 1 & 0.2361 & 0.0094 & 0.89 & 0.26 & 2.384 & 0.373 \\
\hline NGC 0470 & $\mathrm{Sb}$ & 2150 . & 200 & 143 & & 2 & 0.0993 & 0.0136 & 2.11 & 0.15 & 1.427 & 0.295 \\
\hline NGC 0532 & Sab & 2440 . & & 124 & 0.208 & 1 & 0.1687 & 0.0107 & & & 2.942 & 0.432 \\
\hline NGC 0691 & $\mathrm{Sbc}$ & 2867 . & 202 & 108 & 0.496 & 1 & 0.2249 & 0.0089 & & & 2.933 & 0.357 \\
\hline NGC 0918 & $\mathrm{SBc}$ & 1168. & 134 & & $<0$ & 2 & 0.1196 & 0.0207 & 1.18 & 0.42 & 1.836 & 0.859 \\
\hline NGC 0949 & $\mathrm{Sb}$ & 852. & 93 & 69 & 0.177 & 2 & 0.0774 & 0.0073 & 2.36 & 0.42 & 1.554 & 0.236 \\
\hline NGC 1024 & Sab & 3527. & 244 & 173 & & 2 & 0.2368 & 0.0049 & 0.77 & 0.16 & 2.613 & 0.230 \\
\hline NGC 1056 & $\mathrm{Sa}$ & 1272 . & & 77 & 1.041 & 2 & 0.1018 & 0.0045 & 3.45 & 0.23 & 1.552 & 0.174 \\
\hline NGC 1137 & $\mathrm{Sb}$ & 3032. & 151 & 79 & 0.408 & 2 & 0.1431 & 0.0057 & 0.67 & 0.22 & 2.101 & 0.208 \\
\hline NGC 1169 & $\mathrm{SBb}$ & 2316 . & 248 & 148 & 0.562 & 2 & 0.2520 & 0.0070 & & & 2.681 & 0.250 \\
\hline NGC 1171 & $\mathrm{Sc}$ & 4964. & 132 & 84 & 0.151 & 1 & 0.1707 & 0.0083 & 0.52 & 0.24 & 2.285 & 0.338 \\
\hline NGC 1175 & S0-a & 4963. & & 229 & & 2 & 0.2897 & 0.0048 & 0.08 & 0.04 & 2.561 & 0.159 \\
\hline NGC 1186 & SBbc & 4964 . & & 139 & & 1 & 0.1694 & 0.0091 & 2.91 & 0.33 & 2.065 & 0.371 \\
\hline NGC 1209 & $\mathrm{E}$ & 2687. & & 237 & & 1 & 0.2773 & 0.0057 & & & 2.105 & 0.183 \\
\hline NGC 1343 & $\mathrm{SBb}$ & 2503 . & 65 & 128 & & 2 & 0.2277 & 0.0184 & 1.04 & 0.24 & 2.631 & 0.210 \\
\hline NGC 1485 & $\mathrm{Sb}$ & 1565. & 135 & 68 & 0.206 & 3 & 0.1608 & 0.0086 & & & 2.159 & 0.463 \\
\hline NGC 1726 & So & 4035. & & 247 & & 2 & 0.2735 & 0.0069 & & & 2.062 & 0.288 \\
\hline NGC 2146 & SBab & 1526. & 190 & 121 & $<0$ & 2 & 0.0798 & 0.0062 & 4.19 & 0.16 & 0.917 & 0.269 \\
\hline NGC 2339 & $\mathrm{SBbc}$ & 2668 . & 214 & & 0.150 & 2 & 0.1712 & 0.0355 & 1.29 & 0.26 & 1.660 & 0.710 \\
\hline NGC 2365 & $\mathrm{SBa}$ & 2732 . & 206 & 121 & 0.582 & 1 & 0.1992 & 0.0069 & 1.71 & 0.18 & 2.630 & 0.258 \\
\hline NGC 2403 & $\mathrm{SBc}$ & 400 & 112 & & & 1 & 0.0685 & 0.0095 & & & 1.505 & 0.407 \\
\hline NGC 2523 & $\mathrm{SBbc}$ & 3861. & 254 & 141 & & 1 & 0.2154 & 0.0069 & 0.81 & 0.13 & 2.626 & 0.257 \\
\hline NGC 2543 & $\mathrm{SBb}$ & 2894. & & & & 2 & 0.1129 & 0.0103 & 1.44 & 0.14 & 1.738 & 0.245 \\
\hline NGC 2545 & SBab & 5026 . & 222 & 179 & & 2 & 0.2296 & 0.0131 & & & 2.599 & 0.144 \\
\hline NGC 2549 & S0 & 1811. & & 145 & & 2 & 0.2515 & 0.0079 & 0.19 & 0.07 & 2.723 & 0.112 \\
\hline NGC 2633 & $\mathrm{SBb}$ & 2536 . & 149 & 99 & & 2 & 0.0906 & 0.0042 & 1.36 & 0.16 & 0.922 & 0.135 \\
\hline NGC 2648 & $\mathrm{Sa}$ & 2578 . & & 161 & 2.782 & 2 & 0.2423 & 0.0055 & & & 2.511 & 0.185 \\
\hline NGC 2712 & $\mathrm{SBb}$ & 2362 . & 164 & 96 & & 2 & 0.1490 & 0.0058 & 1.25 & 0.13 & 2.063 & 0.161 \\
\hline NGC 2715 & $\mathrm{SBc}$ & 1778 . & 165 & & $<0$ & 2 & 0.1015 & 0.0218 & 2.59 & 1.40 & 1.780 & 0.437 \\
\hline NGC 2726 & $\mathrm{Sa}$ & 1689. & & 82 & $<0$ & 1 & 0.1785 & 0.0088 & 1.60 & 0.28 & 2.276 & 0.370 \\
\hline NGC 2732 & S0 & 1867. & & 154 & & 4 & 0.2215 & 0.0075 & 0.50 & 0.12 & 2.401 & 0.193 \\
\hline NGC 2742 & $\mathrm{Sc}$ & 1689. & 157 & 66 & $<0$ & 2 & 0.1508 & 0.0075 & 0.83 & 0.19 & 2.031 & 0.316 \\
\hline NGC 2798 & $\mathrm{SBa}$ & 2225 . & 128 & 113 & & 1 & 0.0687 & 0.0053 & 4.10 & 0.11 & 0.910 & 0.169 \\
\hline NGC 2841 & $\mathrm{Sb}$ & 1024 . & 307 & 210 & 0.605 & 2 & 0.2987 & 0.0046 & 0.67 & 0.14 & 2.420 & 0.094 \\
\hline NGC 2844 & $\mathrm{Sa}$ & 2225 . & 146 & 111 & 0.982 & 1 & 0.1525 & 0.0064 & 1.63 & 0.21 & 2.190 & 0.237 \\
\hline NGC 2894 & $\mathrm{Sa}$ & 2783 . & 200 & 154 & & 2 & 0.2006 & 0.0054 & 0.46 & 0.16 & 2.327 & 0.200 \\
\hline NGC 2916 & $\mathrm{Sb}$ & 4222 . & 228 & 139 & & 2 & 0.1465 & 0.0283 & & & 1.768 & 0.857 \\
\hline NGC 2964 & $\mathrm{SBbc}$ & 2025 . & 152 & 95 & & 2 & 0.1035 & 0.0054 & 2.73 & 0.13 & 1.508 & 0.144 \\
\hline NGC 2976 & $\mathrm{Sc}$ & 341. & 54 & & & 2 & 0.0571 & 0.0151 & 3.75 & 0.53 & 1.378 & 0.467 \\
\hline NGC 2985 & $\mathrm{Sb}$ & 1583. & 200 & 143 & 1.339 & 4 & 0.1967 & 0.0063 & & & 2.200 & 0.088 \\
\hline NGC 3021 & $\mathrm{Sbc}$ & 2030 . & 138 & 58 & 0.059 & 2 & 0.1317 & 0.0076 & 0.73 & 0.15 & 2.111 & 0.300 \\
\hline NGC 3031 & $\mathrm{Sb}$ & 341. & 215 & 159 & & 2 & 0.2616 & 0.0039 & 2.18 & 0.28 & 2.691 & 0.081 \\
\hline NGC 3041 & $\mathrm{SBc}$ & 1776. & 159 & 95 & 0.145 & 2 & 0.1456 & 0.0281 & & & 1.756 & 0.396 \\
\hline NGC 3043 & $\mathrm{Sb}$ & 2863. & 126 & & & 2 & 0.0743 & 0.0110 & 3.16 & 0.28 & 1.576 & 0.562 \\
\hline NGC 3067 & SBab & 2016 . & 110 & 81 & & 2 & 0.0982 & 0.0075 & 1.36 & 0.12 & 1.812 & 0.395 \\
\hline NGC 3169 & $\mathrm{Sa}$ & 1707. & 175 & 167 & & 2 & 0.1489 & 0.0054 & 0.89 & 0.15 & 1.911 & 0.124 \\
\hline NGC 3190 & $\mathrm{Sa}$ & 1872 . & 218 & 178 & & 2 & 0.1882 & 0.0115 & & & 2.044 & 0.218 \\
\hline NGC 3226 & $\mathrm{E}$ & 1872 . & & 190 & & 1 & 0.2648 & 0.0061 & 1.26 & 0.26 & 2.322 & 0.213 \\
\hline NGC 3227 & $\mathrm{SBa}$ & 1872. & 244 & 136 & & 2 & 0.1297 & 0.0047 & 40.40 & 2.23 & 1.005 & 0.132 \\
\hline NGC 3254 & $\mathrm{Sbc}$ & 1935. & 191 & 113 & 0.736 & 2 & 0.2068 & 0.0081 & 2.07 & 0.16 & 2.371 & 0.152 \\
\hline NGC 3294 & $\mathrm{Sc}$ & 2209 . & 202 & 77 & 0.038 & 2 & 0.1585 & 0.0079 & 0.95 & 0.37 & 2.283 & 0.303 \\
\hline
\end{tabular}

profile along the slit and the extent of the object was manually determined (to indicate where the sky background can be measured). This information is stored as FITS keywords in the archived files, and properly carried along the pipeline. Bias subtraction and flat-field corrections are made with high $S / N$ exposures on the illuminated dome and on the twilight sky.

The removal of the spikes due to cosmic rays is critical in our case, since the spectra are under-sampled along the wavelength direction. The procedure used to clip the spikes is described in the README file; we checked manually that it did not produce a significant number of spurious detections and we considered it robust enough to be applied blindly along the automatic reduction pipeline.
The re-sampling of the spectra into wavelength is performed after spike removal. A wavelength calibration relation was computed for each run and we used the arc spectra taken immediately before and after each observation to check for possible wavelength shifts (flexure in the spectrograph).

A constant sky background was calculated at each wavelength and subtracted. Sky subtraction is not critical in the present paper, which is concerned only with the central regions. The sky subtraction is also automatically done along the Hypercat pipeline by subtracting the average of the two sky regions taken on each side of the object. Each sky spectrum is determined as the median, at each wavelength, over the sky region. 
Table 1. continued

\begin{tabular}{|c|c|c|c|c|c|c|c|c|c|c|c|c|}
\hline Object & Type & $V_{\text {dist }}$ & $V_{\text {rot }}^{\mathrm{m}}$ & $\sigma_{0}$ & $R_{6}^{\prime}$ & $N_{\text {obs }}$ & $\mathrm{Mg}_{2}$ & \pm & OIII & \pm & $<\mathrm{Fe}>$ & \pm \\
\hline$(1)$ & $(2)$ & $(3)$ & $(4)$ & $(5)$ & (6) & (7) & $(8)$ & (9) & $(10)$ & $(11)$ & $(12)$ & $(13)$ \\
\hline NGC 3338 & $\mathrm{Sc}$ & 1407. & 186 & 88 & 0.132 & 2 & 0.1862 & 0.0081 & 0.35 & 0.07 & 2.263 & 0.248 \\
\hline NGC 3368 & SBab & 1435. & 199 & 128 & 0.826 & 1 & 0.1839 & 0.0051 & 0.61 & 0.23 & 2.633 & 0.152 \\
\hline NGC 3370 & $\mathrm{Sc}$ & 1691. & 143 & 49 & 0.168 & 2 & 0.1126 & 0.0056 & 0.55 & 0.09 & 1.735 & 0.198 \\
\hline NGC 3430 & $\mathrm{SBc}$ & 2209 . & 177 & & 0.055 & 2 & 0.1446 & 0.0122 & 0.78 & 0.27 & 1.967 & 0.372 \\
\hline NGC 3437 & $\mathrm{SBc}$ & 1707. & 148 & 105 & 0.031 & 1 & 0.1072 & 0.0069 & 1.65 & 0.16 & 1.557 & 0.267 \\
\hline NGC 3627 & $\mathrm{SBb}$ & 796. & 174 & 124 & & 2 & 0.1418 & 0.0042 & 1.45 & 0.13 & 1.983 & 0.099 \\
\hline NGC 3675 & $\mathrm{Sb}$ & 1152. & 223 & 116 & 0.530 & 2 & 0.1838 & 0.0088 & 0.40 & 0.17 & 2.449 & 0.161 \\
\hline NGC 3718 & $\mathrm{SBa}$ & 1406. & 241 & 172 & & 2 & 0.2657 & 0.0064 & 1.83 & 0.29 & 2.173 & 0.205 \\
\hline NGC 3726 & $\mathrm{SBc}$ & 1366. & 157 & 71 & 0.221 & 4 & 0.0853 & 0.0092 & 0.82 & 0.13 & 1.761 & 0.508 \\
\hline NGC 3756 & SBbc & 1391. & 147 & & 0.033 & 1 & 0.1301 & 0.0135 & 0.75 & 0.19 & 2.275 & 0.589 \\
\hline NGC 3810 & $\mathrm{Sc}$ & 1360. & 143 & 71 & 0.214 & 1 & 0.1357 & 0.0061 & 0.42 & 0.20 & 1.978 & 0.236 \\
\hline NGC 3893 & $\mathrm{SBc}$ & 1366. & 148 & 107 & 0.114 & 1 & 0.1187 & 0.0059 & 0.46 & 0.12 & 1.852 & 0.213 \\
\hline NGC 3898 & Sab & 1391. & 282 & 204 & 1.626 & 1 & 0.2924 & 0.0048 & 1.08 & 0.19 & 2.449 & 0.132 \\
\hline NGC 4237 & SBbc & 1360. & 137 & 65 & & 1 & 0.1359 & 0.0072 & 0.46 & 0.22 & 1.827 & 0.277 \\
\hline NGC 4258 & SBbc & 756. & 205 & 134 & 0.545 & 2 & 0.1775 & 0.0044 & 5.01 & 0.36 & 2.250 & 0.122 \\
\hline NGC 4303 & SBbc & 1360. & 151 & 109 & & 2 & 0.1508 & 0.0039 & 2.28 & 0.34 & 1.989 & 0.116 \\
\hline NGC 4380 & Sab & 1360. & 143 & 63 & & 1 & 0.1560 & 0.0088 & & & 2.579 & 0.357 \\
\hline NGC 4501 & $\mathrm{Sb}$ & 1360. & 277 & 163 & 0.208 & 2 & 0.2360 & 0.0049 & 1.80 & 0.20 & 2.724 & 0.140 \\
\hline NGC 4579 & $\mathrm{SBb}$ & 1360. & 264 & 158 & & 1 & 0.1970 & 0.0052 & 1.92 & 0.35 & 2.264 & 0.156 \\
\hline NGC 4698 & $\mathrm{Sa}$ & 1360. & 243 & 133 & & 1 & 0.2344 & 0.0053 & 1.47 & 0.20 & 2.534 & 0.162 \\
\hline NGC 4725 & SBab & 1360. & 241 & 143 & 1.289 & 1 & 0.2731 & 0.0048 & 1.67 & 0.25 & 2.995 & 0.131 \\
\hline NGC 4826 & Sab & 644. & 149 & 99 & 0.216 & 1 & 0.1817 & 0.0060 & 1.36 & 0.22 & 2.250 & 0.206 \\
\hline NGC 5033 & $\mathrm{Sc}$ & 1341. & 211 & 133 & & 2 & 0.1955 & 0.0056 & 3.43 & 0.19 & 2.386 & 0.132 \\
\hline NGC 5055 & Sbc & 709. & 211 & 110 & 0.589 & 2 & 0.2216 & 0.0042 & 0.60 & 0.20 & 2.687 & 0.089 \\
\hline NGC 5364 & $\mathrm{Sbc}$ & 1070. & 171 & 91 & & 1 & 0.1837 & 0.0161 & & & 1.578 & 0.718 \\
\hline NGC 7177 & $\mathrm{SBb}$ & 1451. & 170 & 126 & $<0$ & 1 & 0.1771 & 0.0060 & & & 2.507 & 0.209 \\
\hline NGC 7331 & $\mathrm{Sbc}$ & 910. & 251 & 137 & 1.076 & 2 & 0.2044 & 0.0051 & & & 2.337 & 0.113 \\
\hline NGC 7606 & $\mathrm{Sb}$ & 2159. & 275 & 147 & & 1 & 0.2397 & 0.0086 & & & 2.580 & 0.337 \\
\hline NGC 7678 & $\mathrm{SBc}$ & 3346. & 179 & 107 & & 2 & 0.1024 & 0.0116 & 1.59 & 0.25 & 1.463 & 0.363 \\
\hline NGC 7814 & Sab & 987. & 231 & 170 & & 2 & 0.2180 & 0.0061 & 0.15 & 0.04 & 2.137 & 0.222 \\
\hline UGC 02906 & $\mathrm{Sb}$ & 2581. & & 148 & 0.347 & 1 & 0.2316 & 0.0110 & 1.43 & 0.31 & 1.716 & 0.453 \\
\hline UGC 03580 & $\mathrm{SBa}$ & 1547. & 103 & & 0.595 & 3 & 0.0709 & 0.0141 & 2.14 & 0.55 & 1.323 & 0.231 \\
\hline UGC 03828 & $\mathrm{SBb}$ & 3651. & 125 & 103 & & 2 & 0.1136 & 0.0115 & & & 1.561 & 0.210 \\
\hline \multicolumn{13}{|c|}{ Additional objects } \\
\hline Gal. bulge & Sbc & & 220 & 150 & & 0 & 0.2590 & 0.0300 & & & 2.720 & 0.310 \\
\hline M 31 & $\mathrm{Sb}$ & 50. & 249 & 170 & & 0 & 0.2650 & 0.0020 & & & 2.875 & 0.300 \\
\hline M 33 & Sbc & 56. & 95 & 30 & & 0 & 0.0140 & 0.0050 & & & 1.410 & 0.150 \\
\hline M 104 & $\mathrm{Sa}$ & 1172. & 362 & 243 & & 0 & 0.3450 & 0.0090 & & & 3.190 & 0.300 \\
\hline
\end{tabular}

Notes: Column 1 is the galaxy identifier (coordinates are given in the electronic version of the Table). Column 2 is the morphological type taken from the PGC96 catalog (Paturel et al. 1997). Column 3 is the distance expressed in km s $\mathrm{s}^{-1}$ computed from a group velocity and Great-Attractor model as in Prugniel et al. (1999). Column 4 is the maximum velocity of rotation from PGC96, in $\mathrm{km} \mathrm{s}^{-1}$. It is deduced from Hi line-width corrected for inclination and internal dispersion. Column 5 is the central velocity dispersion from Hypercat, in $\mathrm{km} \mathrm{s}^{-1}$. Column 6 is the $R_{6}^{\prime}$ radius, as defined in Sect. 5.1, in $h^{-1} \mathrm{kpc} ; R_{6}^{\prime}<0$ means that the disk contamination is important at any radius. Column 7 is the number of individual observations. Columns 8 and 9 are the fully reduced $\mathrm{Mg}_{2}$ index, normalized within an aperture of $0.2 h^{-1} \mathrm{kpc}$ and its associated error, in magnitudes. Columns 10 and 13 are the [OIII] $\lambda 5007 \AA$ equivalent width measured by a Gaussian fit, and its associated error, in $\AA$. Columns 12 and 13 are the $\langle\mathrm{Fe}>$ index and its error, in magnitudes (the Fe 5270 and Fe 5335 indices are available in the electronic version of this Table). The line-strength indices for the Local-Group galaxies and for NGC 4594 are taken from Hypercat. The maximum velocity of rotation of the Milky Way comes from Fich \& Tremaine (1991).

The small number of flux calibrators taken during our observations (the initial goal was to study the kinematics) does not allow the monitoring of changes in the atmospheric absorption. Thus, we calibrated all the observations using a flux-calibration relation, modelled by a second-degree polynomial, obtained by comparing the spectrum of SAO 055164 from the Jacoby (1984) stellar library (referenced as: fa:L1984JACO/00097 in Hypercat) with the observation fa:L93011HP1/00013. We have checked that this relation is consistent with the calibration obtained with spectra from the Jones library (Leitherer et al. 1996), as done in Golev et al. (1999: hereafter GPSL99).

The extraction of the 1D spectra was done by averaging the five central scans of each image; the resulting $S / N$ is generally above 30 per $\AA$. The extracted scanspectra were individually inspected and the FITS spectra are available at the journal archive (CDS) together with more details about the extraction in the README file, as well as instructions for re-doing the extraction from the public HFA with customized parameters (e.g. changing the aperture radius).

\subsection{Measurements of the spectrophotometric indices}

The raw values of magnesium indices, $\left(\mathrm{Mg}_{2 \text { obs }}\right)$, were measured according to the Lick definition (see Worthey et al. 1994) after the extracted spectra where convolved by a Gaussian to reduce their spectral resolution to the Lick system (8.4 $\AA$ for $\mathrm{Mg}_{2}$, Worthey \& Ottaviani 1997).

The central wavelength band of $\mathrm{Mg}_{2}$ (5154.125 $5196.625 \AA)$ and side-bands $(4895.125-4957.625 \AA$ and $5301.125-5366.125 \AA$ ) avoid the [OIII] line $\lambda \simeq 5007 \AA$ 
Table 2. List of observations

\begin{tabular}{lllll}
\hline Dataset name & Date & Paper & $N_{\text {spec }}$ & $N_{\text {obj }}$ \\
\hline L93011HP1 & 1993 January & H1 & 12 & 6 \\
L93021HP1 & 1993 February & H1 & 54 & 28 \\
L93121HP1 & 1993 December & H2 & 28 & 19 \\
L94021HP1 & 1994 February & H2 & 29 & 19 \\
L94121HP1 & 1994 December & H2 & 20 & 7 \\
L95012HP1 & 1995 January & H2 & 12 & 11 \\
\hline
\end{tabular}

Notes: Column 1: Dataset identifier as used in Hypercat. Column 2: Date of observation. Column 3: Reference to the paper where the kinematics are presented, H1: Héraudeau \& Simien (1998); H2: Héraudeau et al. (1999). Column 4: Number of science spectra. Column 5: Number of observed objects.

which is often present in emission in our spectra, but the [OIII] $\lambda \simeq 4959 \AA$ and $[\mathrm{NI}] \lambda \simeq 5199 \AA$ are respectively close to the blue side band and central band. They can possibly contaminate the $\mathrm{Mg}_{2}$ index (Goudfrooij \& Emsellem 1996: hereafter GE96). For this reason, we measured the $[\mathrm{OIII}] \lambda 5007 \AA$ equivalent width which can be used to correct $\mathrm{Mg}_{2}$ for the [NI] emission assuming a constant $[\mathrm{OIII}] /[\mathrm{NI}]$ ratio (GE96 give $[\mathrm{OIII}] /[\mathrm{NI}]=2.7$ ). The [OIII] $\lambda 5007 \AA$ equivalent width was determined from a Gaussian fit with a constant baseline, the reported errors are the formal fitting errors (which may underestimate the real errors). There is no strong contamination of the iron indices by nebular emission.

Our $\mathrm{Mg}_{2}$ measurement procedure was validated using seven stellar spectra provided for this purpose by G. Worthey ${ }^{3}$ together with the measured $\mathrm{Mg}_{2}$.

The reduction of the spectra to the rest frame was done using the cataloged heliocentric velocities (in general from Hi observations). We did this because the Fourier-fitting procedure was not yet available in our reduction pipeline, but we checked afterwards (see below) that this does not induce significant error.

The whole process of spectrum extraction and index measurement was checked against GPSL99. For 136 objects available in HFA, the mean difference on $\mathrm{Mg}_{2}$ (our measurements minus Golev's), $-0.003 \pm 0.001 \mathrm{mag}$, is within the precision quoted in GPSL99. Note that though we are using the same spectra, we did not use the same procedure for, in particular, sky subtracting).

The raw measurements for each of our observations are given in Table 3, available in electronic form only, which lists the values of the $\mathrm{Mg}_{2}$, Fe 5270 and Fe 5335 indices, and the $[\mathrm{OIII}]$ equivalent width.

\subsection{Internal errors}

As discussed in GPSL99, the error on the measured $\mathrm{Mg}_{2}$ index is essentially due to the propagation of the random noise attached to the observed spectra and to the uncertainties on sky subtraction and flux calibration. The different sources of error are reviewed below.

The error resulting from the propagation of observation noise is calculated as explained in Cardiel et al. (1998)

\footnotetext{
3 http://199.120.161.183/worthey
}

except that, instead of using the mean signal-to-noise ratio, $\langle S / N\rangle$, we used the inverse of $\left\langle(S / N)^{-1}\right\rangle$. The reason is that the approximation used in Cardiel et al. increases the sensitivity to "hot" pixels without providing a real simplification.

The $1 \sigma$ error is typically $0.007 \mathrm{mag}$ for $\mathrm{Mg}_{2}$ and $0.3 \AA$ for the iron indices (the $S / N$ of spectra is 30 per $\AA$ ).

Because the spectral resolution is much higher than the Lick resolution, the wavelength re-sampling is not critical to our data reduction (even though the original data are at the limit of under-sampling). We measured the error induced on $\mathrm{Mg}_{2}$ by the re-sampling from pixel to wavelength by shifting the spectra back and forth, by a fraction of a pixel (double re-sampling). The induced error is always smaller than $10^{-5}$ mag for $\mathrm{Mg}_{2}$, i.e. absolutely negligible. This conclusion also holds for the Fe indices.

In order to find the uncertainty induced by the skysubtraction process, we created, for several spectra, copies with different sky levels reflecting the uncertainty on the calculated sky background. We measured $\mathrm{Mg}_{2}$ on the copies and found that the rms deviation was 0.004 mag. The error on both iron indices is $0.1 \AA$.

We determined the error due to flux calibration, according to GPSL99, by comparing the index values obtained with response functions derived using different template stars. Assuming that the distribution of these calibration functions is representative of changes in the flux calibration during the whole run, we found the mean corresponding error to be $\lesssim 0.002 \mathrm{mag}$ on $\mathrm{Mg}_{2}$ and $\lesssim 0.01 \AA$ on Fe.

Using the cataloged heliocentric velocity to reduce the spectra to rest frame induces an error because (i) the apparent velocity differs form the heliocentric velocity by the effect of aberration, and (ii) the spectra are, in addition, affected by shifts due to spectrograph flexure that we did not correct. The standard deviation of the difference between the cataloged heliocentric velocity and the measured velocity of the [OIII] $\lambda 5007 \AA$ line is about $50 \mathrm{~km} \mathrm{~s}^{-1}$ (this is consistent with the typical magnitude of the wavelength lag due to spectrograph flexure, $\leq 1 / 3 \mathrm{px}$, and use of heliocentric velocity). We evaluated the incidence of this error on the measured $\mathrm{Mg}_{2}$ as 0.0006 mag. The sensitivity of Fe to the errors on rest-frame velocity is higher than for $\mathrm{Mg}_{2}$, but the resulting error can still be neglected. These errors are much smaller than the noise error, hence justifying the use of the cataloged heliocentric velocity.

The total internal error for each index is finally the quadratic sum of the errors due to: the noise, the sky subtraction, and the flux calibration. These errors are reported in Table 3.

\subsection{Internal comparisons}

In general, two spectra of the same galaxy were taken at the same telescope pointing, separated by an arc spectrum. Between these observations, the atmospheric 
conditions (flux calibration) are likely comparable (we did not keep information related to atmospheric conditions in the FITS keywords). The uncertainty on the determination of the sky is mostly due to unexplained changes in the response along the slit, resulting in a wavy residual pattern in the sky brightness at the level of about $1 \%$; this is probably due to flexure in the spectrograph and this is strongly correlated between consecutive observations. Hence, the pairwise comparison of $\mathrm{Mg}_{2}$ is supposed to reflect the random noise only.

The mean standard deviation between consecutive spectra of the same galaxies is $0.0083 \mathrm{mag}$ (computed on 40 comparisons), i.e. the error attached to each individual measurement is $0.0059 \mathrm{mag}$. For the same set of observations, the mean random-noise error estimated from $S / N$ is in perfect agreement. Thus we are led to trust our error analysis. The result is similar for the iron indices.

An additional estimate of the uncertainty can be derived from the three galaxies with measurements repeated in another run (NGC 2985, NGC 7331, and UGC 03580); for these, the rms errors on the zero-point of a single run are $0.013 \mathrm{mag}$ for $\mathrm{Mg}_{2}$, and 0.219 and $0.181 \AA$ for Fe 5270 and Fe 5335, respectively. Although of low statistical weight, these values are not incompatible with those quoted below, in Sect. 4.1.

\section{Final measurements and estimation of external error}

The last steps in the measurement process are to combine the different individual raw measurements, to normalize them to a standard aperture and reduce them to a null velocity dispersion.

\subsection{Final $\mathrm{Mg}_{2}$, Fe 5270 and Fe 5335 indices and associated errors}

The final indices for each galaxy were determined as the mean of the different individual measurements weighted by the inverse of the square of the internal error.

The final errors combine the dispersion of the individual measurements and the errors on these measurements; it is on average $0.009 \mathrm{mag}$ on $\mathrm{Mg}_{2}$, and $0.3 \AA$ on Fe 5270 and $\mathrm{Fe} 5335$. In addition to the LICK indices, we also determined the "combined" Fe index $<\mathrm{Fe}>=(\mathrm{Fe} 5270+$ Fe 5335)/2.

The [OIII] equivalent widths are also averaged, weighting the individual values by the error on $\mathrm{Mg}_{2}$ which is directly associated with the $S / N$ ratio of the spectra. This gives a better estimate of the true error than the formal fitting error.

\subsection{Normalization to a standard aperture}

In general, galaxies display radial gradients of spectrophotometric indices (and of other characteristics). So, to study scaling relations or to compare measurements it is necessary to "reduce" these indices to a common reference.
Measurements can be normalized to a "central" value or to an integrated (or asymptotic) value. They can also be given at an isophotal level or ideally at a "scale-free" fiducial radius, as for example the effective radius.

The problem with these reductions is that they are model-dependent, and in general need some extra parameters on the galaxies (e.g., a radius determined from images). In particular, it would be unrealistic to attempt a reduction to the center because gradients become very important near the center and show a wide diversity (Henry \& Worthey 1999; Peletier et al. 1999). A reduction to an asymptotic value also is uncertain.

We call "central" $\mathrm{Mg}_{2}$ the index measured within a small aperture centered on the galaxy. For early-type galaxies the usual choice is to reduce the measurement to a metric aperture (Jørgensen et al. 1995: hereafter JFK95; Golev \& Prugniel 1998) whose diameter is equivalent to $3.4^{\prime \prime}$ at the distance of the Coma cluster $\left(1.19 h^{-1} \mathrm{kpc}\right.$, where $h$ is the usual dimensionless Hubble constant: $h=$ $H_{0} / 100 \mathrm{~km} \mathrm{~s}^{-1} \mathrm{Mpc}^{-1}$ ), though such an aperture encompasses the whole object for galaxies as small as M 32 . The choice of this aperture is suggested by the fact that it matches the typical observed aperture (the correction is on average close to zero). This definition is not "scalefree" and may, in principle, induce spurious correlations when studying scaling relations, but no such effect has been reported in the literature. A correction to the effective radius has been attempted in JFK95, but since it relies on independent measurements of this radius, it is rarely used.

In the actual case of bulges of spiral galaxies, the observed gradients are the combination of the intrinsic population gradient in the bulge, plus the effect of the contamination by the disk population. The latter is probably the most important. For this reason it would be incorrect to use the same normalization law as JFK95. JMA96 used the bulge-to-disk decomposition provided by Kent (1985) and Simien \& de Vaucouleurs (1986) to define a radius into which the contamination is $1 / 7$, and they customized their observations, using a drift-scan method, to integrate inside this radius. A different approach was adopted by Goudfrooij et al. (1999) and by Proctor et al. (2000): the disk contamination was minimized by setting the slit of the spectrograph along the minor axis of edge-on galaxies.

In order to determine the $\mathrm{Mg}_{2}$ aperture normalization for our observations, we fitted the relation:

$\operatorname{Mg}_{2}(g, r)=\operatorname{Mg}_{2}\left(g, r_{0}\right)+a \times \log r / r_{0}$.

Where $g$ is an index running through our list of observations and $r$ is the equivalent radius (see JFK95). The free parameters are $a$, the slope of the relation in magnitudes, and $\mathrm{Mg}_{2}\left(g, r_{0}\right)$, the $\mathrm{Mg}_{2}$ index in the reference aperture of radius $r_{0}$. We have chosen $r_{0}=0.2 h^{-1} \mathrm{kpc}$, computed using the flow-smoothed velocity as a distance estimator (Golev \& Prugniel 1998), because it is close to the mean radius for our sample: $0.16 h^{-1} \mathrm{kpc}$ (for the 5 -pixel slit). For our rectangular aperture, $r$ is computed 


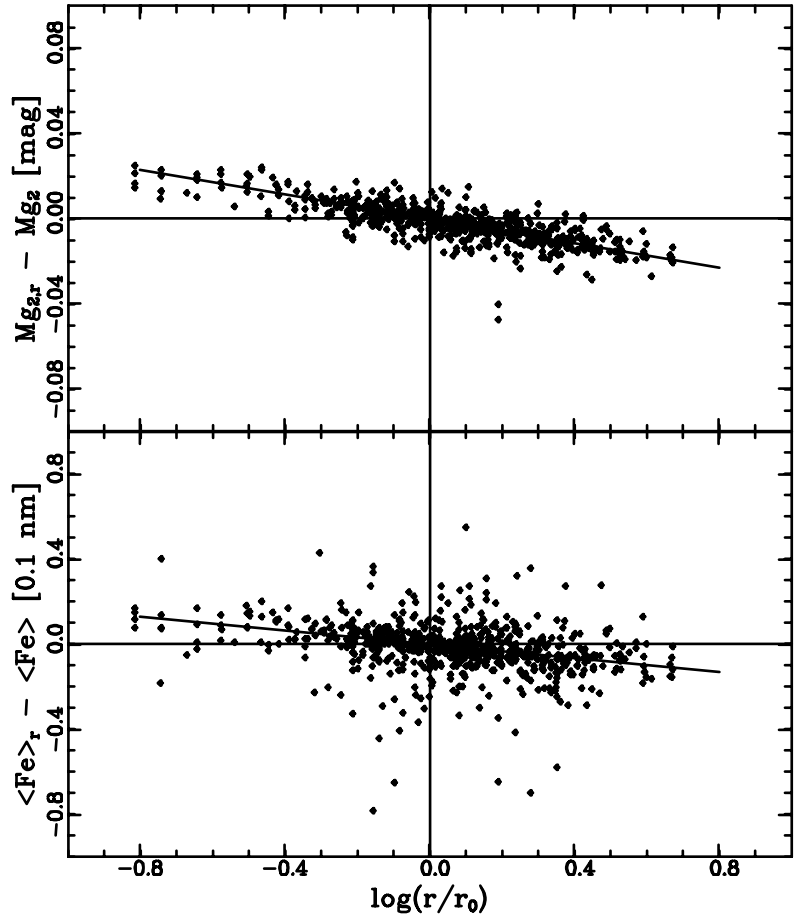

Fig. 1. Aperture normalization relation. In abscissae, the equivalent radius of each measured aperture is normalized to $r_{0}=0.2 h^{-1} \mathrm{kpc}$. Upper panel. The ordinates are the difference between the measured $\mathrm{Mg}_{2}$ and the fitted value of $\mathrm{Mg}_{2}$ in the aperture of radius $r_{0}$ for the galaxy considered. The line is the fitted relation for aperture normalization. Lower panel. The same for the combined iron index $<\mathrm{Fe}>$

as $r=1.025(x y / \pi)^{1 / 2}$, where $x$ and $y$ are the linear dimensions (JFK95). This coefficient depends on both the surface brightness and $\mathrm{Mg}_{2}$ gradients and was determined for elliptical galaxies, using it for bulges is an approximation. For each spectrum we extracted a series of scan spectra corresponding to integration in slit height of, respectively, $y=5,7,11,15$ and 21 pixels. For computing the equivalent radius, we used $x=2.5$ pixels, instead of the real slit width (2 pixels) in order to account for the typical seeing FWHM.

The slope obtained after an ordinary least-squares fit is $a=-0.0290 \pm 0.0014$. The observations of NGC 1169 and NGC 0918 were rejected because the large apertures are strongly contaminated by a bright over-imposed star, as well as the two largest apertures derived from the observation L93021HP1/00320 affected by an unremoved spike. Note that we neglected in the fit the fact that errors on the different measurements for a given observation are not independent.

Figure 1 shows that the quality of the fit is good enough so as not to require a more complicated modelling of the aperture effect.

The slope of the aperture normalization relation is smaller than the one proposed in JFK95 for elliptical galaxies $(-0.04)$. We did not investigate whether this difference is due to the intrinsic nature of the objects (the JFK95 relation is established for elliptical galaxies)

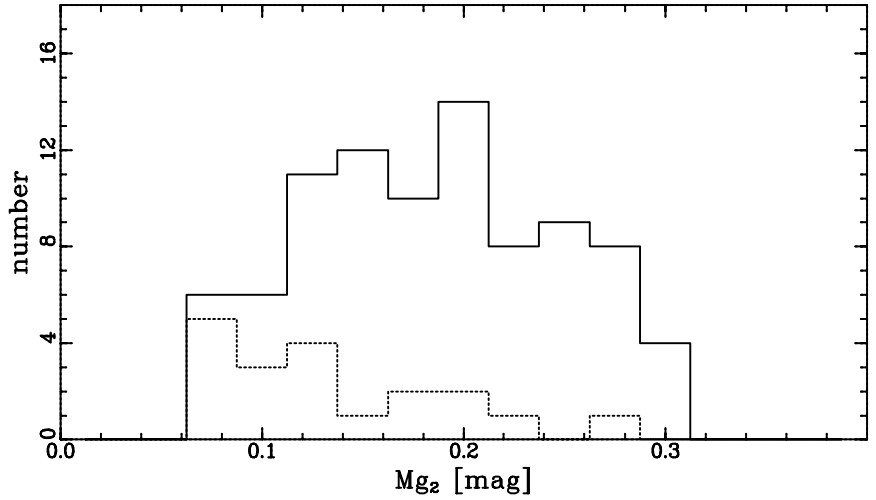

Fig. 2. Distribution of $\mathrm{Mg}_{2}$ for our sample. The full line corresponds to the whole sample, and the dashed line to the galaxies with a measured [OIII] $\lambda 5007 \AA$ equivalent width larger than $2 \AA$

or to properties of the sample (their relation is based on a "model galaxy" elaborated from an inhomogeneous sample of only 51 elliptical galaxies, whereas we used direct measurements on our spectra).

There is evidence that the Fe indices in bulges also display radial gradients (Beauchamp \& Hardy 1997; Goudfrooij et al. 1999; Fisher et al. 1996 for bulges of S0 galaxies). Hence, we searched for a relation similar to that of $\mathrm{Mg}_{2}$. We found $a_{\mathrm{Fe}} 5270=-0.19 \pm 0.04$ and $a_{\mathrm{Fe}} 5335=-0.21 \pm 0.04$. As shown in Fig. 1, the relation is more scattered than for $\mathrm{Mg}_{2}$, but the systematic aperture effect is still very clear.

\subsection{Correction for velocity dispersion}

The spectrophotometric indices are sensitive to the broadening of the spectra by the line-of-sight velocity distribution. The effect is small for $\mathrm{Mg}_{2}$ (because the bandwidth is large) but important for Fe. Hence a correction is necessary to reduce the measurements to a null velocity dispersion. To achieve this, we used the tabulated data of Longhetti et al. (1998). These corrections are slightly smaller $(\approx 10 \%)$ than those of Kuntschner (2000: hereafter K2000), and in complete agreement with those of Trager (1998). The mean correction is $0.002 \mathrm{mag}$ on $\mathrm{Mg}_{2}$ and $0.2 \AA$ on Fe. This correction does not significantly contribute to the final error. For the few galaxies for which the velocity dispersion was not measured, we assumed $\sigma_{0}=$ $100 \mathrm{~km} \mathrm{~s}^{-1}$.

The fully reduced $\mathrm{Mg}_{2}$, Fe 5270, Fe 5335 and $<\mathrm{Fe}>$ indices are presented in Table 1, and Fig. 2 presents the histogram of the $\mathrm{Mg}_{2}$ distribution (Fe 5270 and $\mathrm{Fe} 5335$ are only available in the electronic version of Table 1 ). The galaxies with strong emission are plotted separately in Fig. 2 in order to give a qualitative idea of the effect of a young population on $\mathrm{Mg}_{2}$ : most of the galaxies with $\mathrm{Mg}_{2}<0.1$ mag display nebular emission. 


\subsection{Estimation of external precision}

There are $\mathrm{Mg}_{2}$ measurements of 14 of the galaxies of our sample in Hypercat (see also Golev \& Prugniel 1998). The mean difference between our measurements and Hypercat (ours minus Hypercat) is $0.006 \mathrm{mag}$, with an rms of 0.019. Taking into account the fact that the Hypercat measurements are normalized to an aperture of $0.59 h^{-1} \mathrm{kpc}$, the systematic difference reduces to $0.003 \mathrm{mag}$, and is negligible. The dispersion is compatible with the reported errors.

\section{Discussion}

In this section we will use our spectrophotometric measurements and the other data gathered in Table 1 to study the scaling relations of the bulges and the relations between bulge and disk properties. We will address the following questions:

(1) How far can we consider these indices as characterizing the stellar population of the bulges? Or, what is the magnitude of the contamination by gas and by the stellar population of the disk?

(2) How do our new results constrain the scenarios of bulge formation?

\subsection{Nebular and disk contamination}

The interpretation of the observational relations involving the spectrophotometric indices is not straightforward as they may be contaminated by nebular emission or by the disk population overimposed on the line of sight. In this section, we check the influence of these effects on our indices.

To estimate the level of contamination by disk stars, JMA96 defined the radius $R_{6}$ within which the integrated light contribution of the disk is $1 / 6$ of that of the bulge, and they adapted their observational setup to observe in an aperture equivalent to $R_{6}$. In our case, we also could change the slit height to maintain a constant level of contamination, but this would lead to a very elongated shaperatio of the aperture of integration. We have preferred to use a fixed aperture and to check the level of contamination in this aperture. For this purpose, we determined a $R_{6}^{\prime}$ radius with a definition close to that of $R_{6}$, using the bulge/disk decomposition from H96 available for 46 galaxies of our sample. The H96 decomposition is based on a fit to the $I$-band surface-brightness distribution. The bulge and disk are modelled, respectively, by a Sérsic and an exponential profile, with constant and identical orientation and with constant flattening. We defined $R_{6}^{\prime}$ by: $g_{\mathrm{b}}\left(R_{6}^{\prime}\right) / g_{\mathrm{d}}\left(R_{6}^{\prime}\right)=6$, where $g_{\mathrm{b}}$ and $g_{\mathrm{d}}$ are respectively the growth curves for the bulge and for the disk. The growth curves give the flux integrated in an aperture parameterized by the equivalent radius, i.e. a geometric average of major and minor axes. The definitions of $R_{6}$ and $R_{6}^{\prime}$ formally differ but the values are close, in practice. The values of $R_{6}^{\prime}$ are reported in Table 1 .
The relevance of the $I$ band for the calculation of the central disk contribution deserves a comment. It is well known that photometric decomposition is wavelengthdependent, with an increased relative brightness of the young disk toward blue bandpasses; red bandpasses provide a clearer picture of the axisymmetric, old-star luminosity distribution, due to their lower sensitivity to spiral arms and to extinction by dust. In order to investigate this, we have used the results of De Jong (1996), who performed six-color determinations of bulge and disk parameters on 86 face-on spirals. From these, we extracted a subsample of $42 \mathrm{Sbc-Scd}$ galaxies, expected to be more sensitive to the present problem than earlier types. We calculated the mean difference between the bulge and disk central surface brightnesses, first in $V$, the bandpass closest to the wavelength range of our spectra, and then in $B$ and $I$ : all three averages fall within 0.15 mag of each other. This means that the azimuthally-averaged disk distributions are not very wavelength-dependent; therefore, although the superimposed blue spiral arms are essentially non-axisymmetric, there is reasonable confidence that their difference between $V$ and $I$ is quite limited.

For six galaxies in our sample (NGC 918, NGC 2146, NGC 2715, NGC 2726, NGC 2742 and NGC 7177), $R_{6}^{\prime}$ could not be determined, and in these cases the value given in Table 1 is $R_{6}^{\prime}<0$. There are two causes for this lack of determination. NGC 918, NGC 2715 and NGC 2742 are late-type (Sc) objects with a low-luminosity bulge and the disk contributes to more than $1 / 7$ of the flux within any aperture. In NGC 2726 and NGC 7177 the bulge, although luminous, is diffuse (its Sérsic exponent is $n=1$ ) and the disk contamination is severe. NGC 2146 is an intermediate case with a relatively small bulge and $n=1.39$.

The mean value of $R_{6}^{\prime}$ (excluding objects with $R_{6}^{\prime}<0$ ) is $0.50 \pm 0.08 h^{-1} \mathrm{kpc}$, which is significantly larger than our aperture normalization radius of $r_{0}=0.2 h^{-1} \mathrm{kpc}$, in other words the flux contamination is generally lower than for the JMA96 sample.

Note however that in the extreme case where the disk spectra would be featureless (a very young population), a level of contamination as low as $1 / 6$ could lower the $\mathrm{Mg}_{2}$ by about $0.05 \mathrm{mag}$. Such contamination would be five times the error on our measurements and would be comparable to the dispersion around the $\mathrm{Mg}_{2}$ vs. $\sigma_{0}$ relation. If such an effect exists, we would expect a positive correlation between $R_{6}^{\prime}$ and the residuals to the relations in Fig. 3: highly contaminated measurements characterized by a low $R_{6}^{\prime}$ should be found below the relations. We did not find such an effect either on $\mathrm{Mg}_{2}$ or on $\langle\mathrm{Fe}\rangle$, even for the most contaminated objects $\left(R_{6}^{\prime}<0.2 h^{-1} \mathrm{kpc}\right)$.

Finally, the contamination of the spectrophotometric indices by the disk stars is probably lower than the errors on individual measurements. However, the emission lines constitute in principle a second source of contamination.

The [OIII $\lambda 5007$ emission line is usually detected in our spectra, suggesting that the spectrophotometric indices may be affected by nebular contamination (GE96). In particular, the [NI] doublet at $5199 \AA$ is close to the 
red limit of the $\mathrm{Mg}_{2}$ band (at $5196.625 \AA$ ). According to GE96, we can estimate the order of magnitude of this effect by adopting a [OIII]-to-[NI] conversion factor: $[\mathrm{OIII}] /[\mathrm{NI}]=2.7$. For $[\mathrm{OIII}]=3 \AA$ (only 9 objects have stronger emission, excluding NGC 3227), the inferred contamination induces a change in $\mathrm{Mg}_{2}$ of $-0.004 \mathrm{mag}$. Hence, the nebular contamination is also lost in the measurement errors.

In conclusion, although low-level systematic effects may exist, we are confident that our spectrophotometric indices are representative of the stellar population of the bulges.

The above discussion on contaminations notwithstanding, a last question is worth addressing: Since emission lines are often observed in the central region of galaxies (not considering the cases of LINERs), what gas are they actually tracing? Elements of an answer can come from comparing gas and star kinematics in flattened S0 galaxies; although the structure and evolution of spirals and S0s present obvious differences, some qualitative comparisons may be relevant, and the low dust content of S0s favors a clear view of the stellar and gas motions. As an example, we have selected the sample of Fisher (1997), who presents stellar kinematic profiles on 18 S0 galaxies, together with corresponding [OIII] profiles for nine of them. These measurements show (thus confirming previous knowledge) that, in several cases, the [OIII] kinematics presents a wide variety of behaviors; among them: counter-rotation of disk gas, fast rotation along the minor axis, high gas velocity dispersion at the very center, and presence of gas well outside the equatorial plane of an edge-on galaxy. All this indicates that the inner gas is not always the mere continuity of a large-scale cold gas disk rotating with the stars. Studying the evolution of this inner gas would not be straightforward, and this is obviously beyond the scope of the present work. But it is tempting to suggest that star formation may occur, even at a modest rate, in the off-plane gas (or in gas with an off-plane velocity vector) before it eventually reaches a new equilibrium within the equatorial plane; if so, these young stars would likely move along orbits outside the disk. If this also holds for some spirals in our sample, a fraction of the [OIII] emission might in fact reveal the presence of a young population belonging to the bulge, thus contributing to the secular evolution considered in Sects. 1 and 5.3.

\subsection{The observational relations}

The spectrophotometric indices are expected to correlate between each other and to correlate with other characteristics of the bulges. In particular, the relation between $\mathrm{Mg}_{2}$ and $\sigma_{0}$ was found by IPC96 and JMA96 to be as narrow as the one established for elliptical galaxies. The existence of this relation is interpreted as a mass-metallicity relation and is one of the arguments supporting the hypothesis of a common origin for both bulges and elliptical galaxies.
The relations between indices with a degeneracy in age and metallicity (for example $\mathrm{Mg} b$ vs. $\mathrm{Mg}_{2}$ ) can be used to check the consistency of stellar population models and the accuracy of the measurements. For example, K2000, comparing the three $\mathrm{Mg}$ indices, diagnosed a systematic deviation probably due to the Lick fitting functions. We used a comparison between the two iron indices to check for possible erroneous measurements.

In Fig. 3 we present the relations between the spectrophotometric indices characterizing the stellar population of the bulges and the kinematical parameters indicative of the depth of the central potential well (mass of the bulge) and of the total galactic mass. These kinematical parameters are respectively $\sigma_{0}$ (the central velocity dispersion) and $V_{\mathrm{rot}}^{\mathrm{m}}$ (the maximum velocity of rotation deduced from $\mathrm{HI}$ observations). The $\sigma_{0}$ data are extracted from Hypercat and $V_{\text {rot }}^{\mathrm{m}}$ from LEDA. The galaxies where we detected strong [OIII] $\lambda 5007 \AA$ emission (equivalent width greater than $1 \AA$ ) are represented as open symbols while those where we found no or little gas are shown as closed symbols.

Before discussing these correlations we investigate the outliers individually flagged in Fig. 3, with the hope of spotting spurious measurements or physical peculiarities of these galaxies. The lines drawn in Fig. 3 are the ordinary least-squares fits where the dynamical quantity (on the abscissa) is used as the independent variable. The present purpose is not to discuss quantitatively the slope of these relations and we made this choice to be consistent with the usual practice (e.g., Dressler et al. 1987; Colless et al. 1999).

The following galaxies depart from one or the other of these relations by more than three times the standard deviation. NGC 1343 (VII Zw 8) departs from the $V_{\text {rot }}^{\mathrm{m}}$ vs. $\mathrm{Mg}_{2}$ relation. This galaxy is a slow rotator as seen both from the Hi velocity measurement and from the stellar kinematics (see H2) although it is reasonably inclined on the line of sight (about 50 degrees). The two available spectra are of good quality and have the normal aspect of an old stellar population. Since this galaxy does not depart from the $\mathrm{Mg}_{2}$ vs. $\sigma_{0}$ relation, we believe that the slow rotation is a real physical peculiarity. Taylor et al. (1994) have noted non-circular motions in NGC 1343 which also has a small companion at position radec $(1950)=033233.1+722534$. NGC 0470, 2146, 2798 and 2633 (Arp 80) depart from the $V_{\text {rot }}^{\mathrm{m}}$ vs. $\mathrm{Mg}_{2}$ relation and from the $\sigma_{0}$ vs. $\mathrm{Mg}_{2}$ relation by having low values of $\mathrm{Mg}_{2}$. Our spectra are of good quality and measurement errors are not suspected. All these galaxies display strong [OIII] $\lambda 5007 \AA$ emission. Vanzi et al. (1998) present NGC 2633 as a starburst galaxy. In these four cases the departure may be interpreted as a signature of a young stellar population.

The $\mathrm{Mg}_{2}$ vs. $\sigma_{0}$ is as good as that in JMA96, but it is steeper (this will be discussed in the next section). The tighter relation $\mathrm{Mg}_{2}$ vs. $V_{\text {rot }}^{\mathrm{m}}$ is more unexpected. Indeed, $\sigma_{0}$ can be related to the depth of the potential well or escape velocity in the center and can hence characterize 


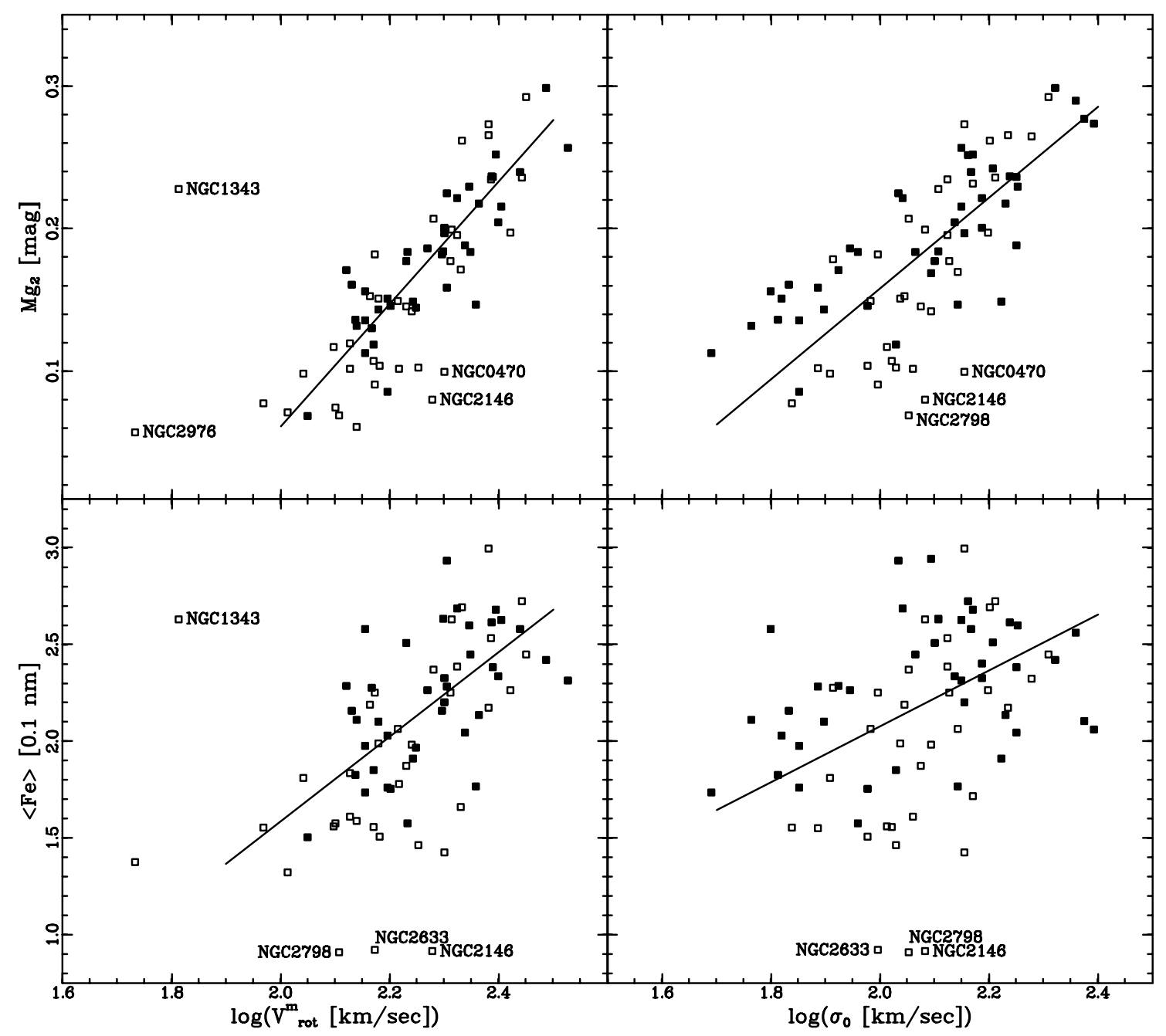

Fig. 3. Top left: $\mathrm{Mg}_{2}$ vs. $V_{\text {rot }}^{\mathrm{m}}$, top right: $\mathrm{Mg}_{2}$ vs. $\sigma_{0}$, bottom left: $<\mathrm{Fe}>$ vs. $V_{\text {rot }}^{\mathrm{m}}$, and bottom right: $<\mathrm{Fe}>$ vs. $\sigma_{0} . V_{\text {rot }}^{\mathrm{m}}$, the maximum rotation velocity (in $\mathrm{km} \mathrm{s}^{-1}$ ) is known for 76 objects and comes from $\mathrm{HI}$ observations (LEDA). $\sigma_{0}$, the central velocity dispersion (in $\mathrm{km} \mathrm{s}^{-1}$ ) is taken from the HYPERCAT database and it is known for 77 objects. Open symbols are for the bulges where the [OIII] equivalent width is greater than $1 \AA$, closed symbols are the other galaxies. The deviant objects are labelled and discussed in the text. The straight lines are least-squares fits using the abscissae as dependent variables; the outlying points were rejected from the fit

both the mass of the galaxy and the concentration of the bulge while $V_{\text {rot }}^{\mathrm{m}}$ is more representative of the mass of the galaxy. Thus it seems that the metallicity of the bulge is rather related to the mass of the galaxy than to the mass of the bulge. In Fig. 4 we present the relations between the spectrophotometric indices and the total absolute magnitude in the $I$ band, $M_{I}$, which is an estimate of the total stellar mass. The correlation is very clear and, using the bulge-disk decomposition from H96, we also found correlations separately with both the magnitudes of the bulge and of the disk; this was not found by JMA96 using the $r$ band magnitudes, and we believe that the difference comes from a more homogeneous bulge-disk decomposition and a larger sample. The use of $I$ band, less sensitive to dust absorption and to a young population, is possibly an additional reason. Similar relations between abundances and mass have also been found: Zaritsky et al. (1994) have found a strong correlation between the disk gas abundance and $V_{\text {rot }}^{\mathrm{m}}$ for spiral galaxies. In the case of S0s, such a relation is confirmed by Fisher et al. (1996).

The galaxies with strong [OIII] $\lambda 5007 \AA$ emission are represented by open symbols in Fig. 3. They generally lie in the lower part of the diagrams, corresponding to the low line-strength indices and low $\sigma_{0}$ or $V_{\text {rot }}^{\mathrm{m}}$. At variance with elliptical galaxies, where the presence of young populations and/or emission lines result in departures almost perpendicular to the relations, these bulges do not dramatically deviate from the general trend. Though the presence of [OIII] may, as well, trace the young population of a LINER, the fact that it is essentially associated with faint bulges leads one to consider an age vs. mass relation. This will be discussed in the next section.

Another new result shown in Figs. 3 and 4 is the strength of the correlations involving $<\mathrm{Fe}>$. Such relations have long been expected (but not observed) for elliptical galaxies (FFI96; Jørgensen 1997, 1999). The < Fe $>$ 


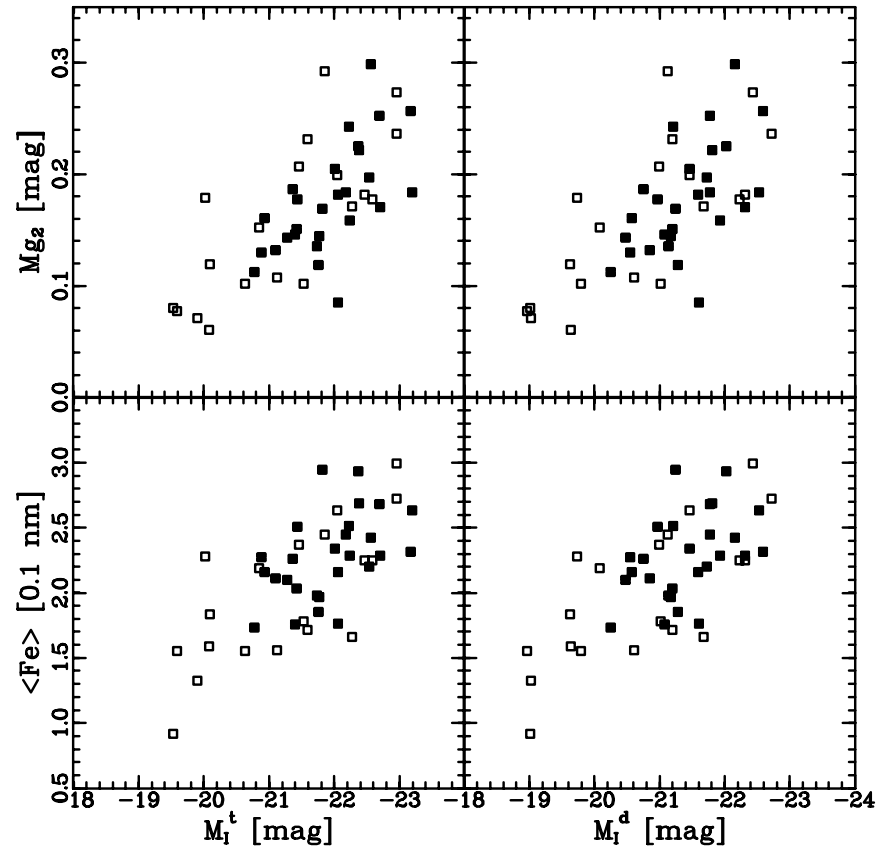

Fig. 4. Relations between indices and $I$-band absolute magnitude. This plot shows the 46 galaxies of our sample with a bulge/disk decomposition in Héraudeau (1996). The distance modulus for absolute magnitudes is calculated using the flowsmoothed velocity given in Hypercat (Golev et al. 1998), with $H_{0}=70 \mathrm{~km} \mathrm{~s}^{-1} \mathrm{Mpc}^{-1} . M_{I}^{\mathrm{t}}$ and $M_{I}^{\mathrm{d}}$ are respectively the total and disk magnitudes. The open symbols represent the bulges where [OIII] $\lambda 5007 \AA>1$

measurements are generally very scattered but, recently, K2000, using high $S / N$ data for 22 galaxies in Fornax, presented convincing evidence for a relation between $\mathrm{Mg}$ and Fe. He interpreted his finding as being dependent on the superior quality of his data $(S / N>30$ per $\AA)$. However, while the $S / N$ of our material is similar, we do not find any correlation between $\mathrm{Mg}_{2}$ and $<\mathrm{Fe}>$ using the same measurement procedure applied to the observations of $87 \mathrm{el}-$ liptical galaxies available in Hypercat (GPSL99). We then conclude that the sensitivity of $<\mathrm{Fe}>$ to $\mathrm{Mg}_{2}$ and mass is a particular property of bulges, in contrast to ellipticals.

The $\mathrm{Mg}_{2}$ vs. $<\mathrm{Fe}>$ diagram is shown in Fig. 5. The over-plotted lines are the evolutive population models given by IPC96. These models are based on the singlepopulation model (SSP) by Borges et al. (1995) and are parameterized by the delay before the onset of Type-Ia $\mathrm{SNe}$, controlling in fine the mean abundance of magnesium relative to iron $([\mathrm{Mg} / \mathrm{Fe}])$. As for elliptical galaxies (O'Connell 1976; Peterson 1976; Faber et al. 1992), this diagram indicates that magnesium is over-abundant (or that iron is deficient), with typically $[\mathrm{Mg} / \mathrm{Fe}]=0.4$. Furthermore, a close examination of Fig. 5 reveals a trend of $[\mathrm{Mg} / \mathrm{Fe}]$ increasing with $\mathrm{Mg}_{2}$ or $\left.<\mathrm{Fe}\right\rangle$, from about 0.2 for $\mathrm{Mg}_{2}=0.1$ to about 0.4 for $\mathrm{Mg}_{2}=0.35$. A similar result is reported for ellipticals by Davies et al. (1993), and Trager et al. (2000b) on Mgb; for S0s by Fischer et al. (1996), and for spiral bulges by JMA96 and IPC96. For ellipticals, Trager et al. consider two classes of scenarios

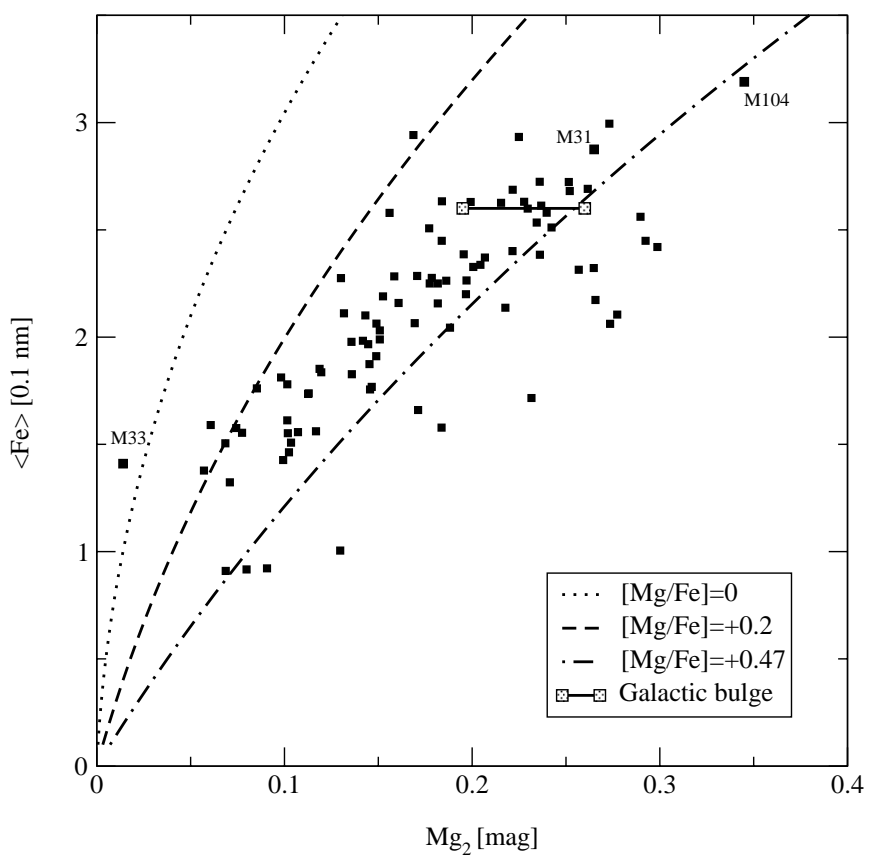

Fig. 5. $\mathrm{Mg}_{2}$ vs. $<\mathrm{Fe}>$. The curves correspond to the IPC96 SSP models with mean abundance ratio $[\mathrm{Mg} / \mathrm{Fe}]=0$ (dotted line), $[\mathrm{Mg} / \mathrm{Fe}]=+0.2$ (dashed line) and $[\mathrm{Mg} / \mathrm{Fe}]=+0.47$ (dash-dotted line). Several objects are shown in addition to our sample; the Galactic-bulge data are from Idiart et al. (1996a) and Delisle (1998)

able to explain such a relation: a variable IMF top, or variation in winds, mass loss, and retention of SN II products as a function of $\sigma_{0}$. For spiral bulges, we have, in addition, the possibility of a complex scenario where a "primordial" population born during the collapse is combined with a "secondary" population formed during evolution.

To summarize, the preliminary conclusions drawn from our observations are: (i) The $\mathrm{Mg}_{2}$ vs. $\sigma_{0}$ (or $\mathrm{Mg}_{2}$ vs. $V_{\text {rot }}^{\mathrm{m}}$ ) relation cannot simply be interpreted as a mass-metallicity dependence. Contrary to elliptical galaxies where the contribution of a "young" population implies deviation from the relation (Prugniel \& Simien 1996), age effects participate in the global $\mathrm{Mg}_{2}$ vs. $\sigma_{0}$ relation for bulges. The young bulges in low-mass galaxies are in the continuity of the massive, old and metal-rich galaxies. (ii) Also contrasting with the elliptical galaxies, the $\langle\mathrm{Fe}\rangle$ index correlates with the other characteristics of the galaxies, namely $\mathrm{Mg}_{2}, \sigma_{0}, V_{\text {rot }}^{\mathrm{m}}$ and the magnitude of the disk. This probably reflects some differences in the process of metal enrichment between ellipticals and bulges.

\subsection{The formation of bulges}

The observational results presented in the previous section support the increasingly accepted hypothesis of intrinsic differences between small and large bulges. However, though it has not been definitely established that a continuity does exist, there are presently no arguments against it. 
In order to account for this assumed continuity, we combine, in Fig. 6a, two stellar populations: a "primary" population formed during the initial collapse and a "secondary" population formed during evolution by the effect of bars or interactions with the environment. The primary population was formed rapidly, probably before the disk, and has been enriched thanks to SN II events. The secondary population may result from a (low) continuous formation process, possibly concentrated in one or several bursts resulting from bar formation-destruction cycles, and/or it may be produced by a starburst triggered by merging or interactions.

We modelled these two basic populations using the SSP models by Borges et al. (1995) which are computed for a range of $[\mathrm{Fe} / \mathrm{H}],[\mathrm{Mg} / \mathrm{Fe}]$ and ages. They are based on a stellar library and on fitting functions and they agree with the models of Weiss (1995) based on synthetic spectra for a mixing-length parameter of about -2 to -2.5 . The primary population is assumed to be 16-Gyr old and very deficient in iron, $[\mathrm{Mg} / \mathrm{Fe}]=0.6$, and to have an iron abundance going from solar to sub-solar $([\mathrm{Fe} / \mathrm{H}]=0$ to -1$)$. We chose an iron deficiency larger than commonly observed in order to account for the fact that the observed $[\mathrm{Mg} / \mathrm{Fe}]$ will combine the primary and secondary populations and hence have a $[\mathrm{Mg} / \mathrm{Fe}]$ lower than the primary population. The secondary population has a solar $[\mathrm{Mg} / \mathrm{Fe}]$, since we assume it results from a slow formation process leaving time for Type-Ia SNe to enrich the gas in iron. Its $[\mathrm{Fe} / \mathrm{H}]$ is equal to that of the primary because the iron enrichment is controlled by the balance between supernova-driven winds and gravity (hence mass); moreover, as an a posteriori justification of this choice, higher $[\mathrm{Fe} / \mathrm{H}]$ values would have led in Fig. 6 (see below) to a model encompassing a wider, unnecessarily empty, upper zone. The age of the secondary population decreases from $16 \mathrm{Gyr}$ for $[\mathrm{Fe} / \mathrm{H}]=0$ to $1 \mathrm{Gyr}$ for $[\mathrm{Fe} / \mathrm{H}]=-1$ (logarithmic dependence). Actually, the age of the secondary does not need to be taken stricto sensus. For the sake of simplicity we are considering SSP models but the real process is an evolution of the SFR and metallicity during its history: an old age for the secondary may as well be understood as an efficient star formation resulting in an early consumption of the gas, and at the opposite extreme, a young age may be interpreted as a low and almost constant SFR. In massive systems, the star formation is efficient while it is less efficient in low-mass objects. This is corroborated by ample evidence, including a simple observational argument: it is well known that large spiral galaxies are significantly dustier than small ones, in terms of extinction as a function of mass.

The sequences corresponding to these models have been computed with Eq. (11) of Borges et al. (1995). The two continuous lines at high and low $\mathrm{Mg}_{2}$, respectively, correspond to the primary and secondary populations. The dashed lines connect the populations corresponding to $[\mathrm{Fe} / \mathrm{H}]=0,-0.5$ and -1 (from high to low $<\mathrm{Fe}>$ ). Since the wavelength regions of $\mathrm{Mg}_{2}$ and $<\mathrm{Fe}>$ are not very distant, these dashed lines are close to the locus of the combined population.
We can distinguish three regions in Fig. 6a, labelled A, $\mathrm{B}$, and $\mathrm{C}$, which we are going to discuss in turn.

\subsubsection{Region A}

This region corresponds to low-metallicity populations and involves a strong contribution from a young secondary: most of the bulges observed in this region display significant ionized gas emission. We note however that a majority of objects (14 out of 23 ) lack a $R_{6}^{\prime}$ value, and therefore we cannot exclude a slightly higher level of contamination by the disk light. However, we also note that 15 galaxies in this region are barred; the previous argument may therefore be reversed: this bar plays an important role in bringing material to the very center (see, e.g., Bonatto et al. 1998), and thus it is most likely contributing to the enrichment of the bulge population.

Not unexpectedly, the literature is scarce in detailed studies on the stellar population of small bulges. As a rare example, we propose M 33, although it is an extreme case, with a vanishingly small bulge: the central light concentration is only $3.5^{\prime \prime}$ in diameter. Davidge (2000) carried out near-infrared observations with an angular resolution of $0.3^{\prime \prime}$; from broad-band and absorption-line indices, he found a metal-poor population $([\mathrm{Fe} / \mathrm{H}] \simeq-1$ compared to the near-solar inner disk); this is surrounding the bluer, younger nucleus. We can identify the former with the bulge primary population; the high-density nucleus, suspected of having experienced a core collapse, could have accreted recent disk material at the expense of the fainter bulge. Indeed, there is evidence for an active starforming activity in the inner disk, 1-3 Gyr ago (Davidge 2000 and references therein). We consider the nucleus-andbulge system as an extreme example of a small bulge.

\subsubsection{Region $B$}

This region follows the primary sequence, and the contribution of the secondary population is minor (or absent).

The bulge of M 104 and, probably, the bulges of the Galaxy and M 31, belong to this region.

For the Galactic bulge, we have given the two measurements by Delisle (1998) and Idiart et al. (1996a) because they are significantly different. Measurements of the integrated index on the Galactic Bulge are complicated by dust absorption and contamination by disk stars. The overwhelming evidence (Wyse 1999 and references therein) for the Galactic Bulge is that its stars are old, except for a flat young component which may be part of the disk. The bulge stars are under-studied, but in the available sample different $\alpha$-elements show different patterns, unexplained within the context of only SNe II enrichment. This may point to the fact that our bulge mostly results from the primary population, with a small contribution from the secondary, i.e. it belongs to region $\mathrm{B}$, or to a region intermediate between $\mathrm{B}$ and $\mathrm{C}$. 


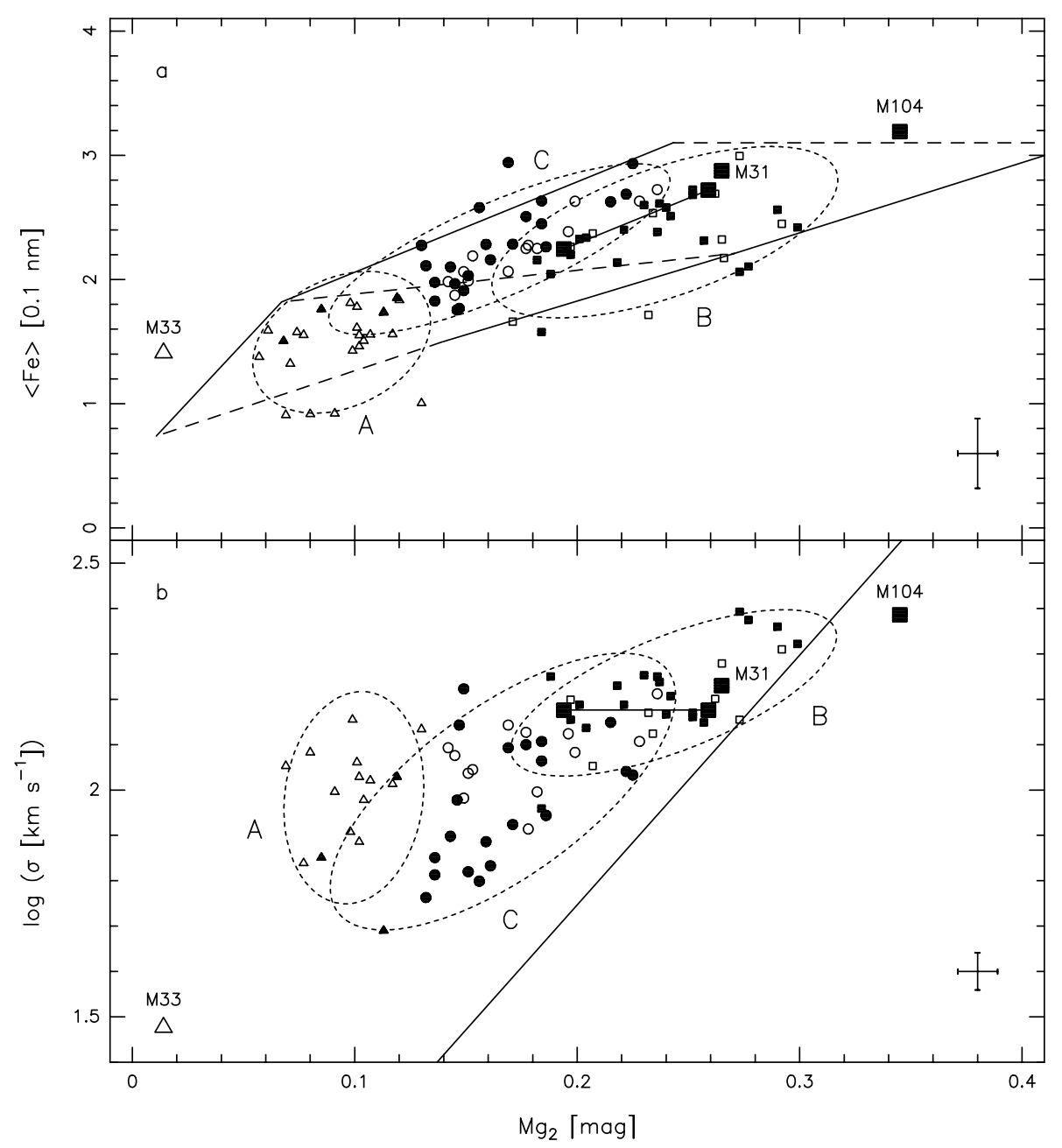

Fig. 6. a) $\mathrm{Mg}_{2}$ vs. $\langle\mathrm{Fe}\rangle$, with loci from models by Borges et al. (1995). Open symbols: galaxies with [OIII] $>1 \AA \AA$ filled symbols: galaxies with less or no [OIII]. Several particular cases are shown as larger filled squares; the unlabelled two, linked by a solid segment, are different determinations of the Galactic bulge (Idiart et al. 1996a; Delisle 1998). The average error bars are shown. Models: the right-hand solid line represents the primary population with $[\mathrm{Mg} / \mathrm{Fe}]=0.6$, from $[\mathrm{Fe} / \mathrm{H}]=0$ (upper end) to -1 (lower end); the left-hand solid line represents the secondary population with $[\mathrm{Mg} / \mathrm{Fe}]=0$, from $16 \mathrm{Gyr}$ and $[\mathrm{Fe} / \mathrm{H}]=0$ (upper end) to $1 \mathrm{Gyr}$ and $[\mathrm{Fe} / \mathrm{H}]=-1$ (lower end). Dashed ellipses represent the boundaries of regions A, B, and C, as calculated by the moments of the member distributions. See Sect. 5.3 for details. b) $\log \sigma_{0} \mathrm{vs}_{\mathrm{Ng}} \mathrm{Mg}_{2}$. Same symbols as in a) The solid line represents the regression for elliptical galaxies (Dressler et al. 1987), slightly corrected as explained in Sect. 5.3

The position of the bulge of M 31 has not been determined precisely: our adopted indices are an average between the measurements by Trager et al. (1998) and those by Sil'chenko et al. (1998). The former is probably more representative of the nucleus while the latter is an average over a region similar to our $0.2 h^{-1} \mathrm{kpc}$ standard aperture. The width of the giant branch in color-magnitude diagrams indicate that this bulge exhibits a rather broad metallicity distribution in its outer parts, but a narrow metallicity distribution interior to $1 \mathrm{kpc}$ (Renzini 1999; Rich 1999).

Both the M 31 and Milky-Way bulges have an old and metal-rich population without clear evidence for important intermediate-age sub-population.

Among the objects lying close to the primarypopulation model, almost all (11 out of 13), are unbarred. This is consistent with the picture of a population mostly shaped by the initial infall, and not by the mixing with the output of the secular evolution of a bar ${ }^{4}$.

In general we did not detect strong emission lines in these bulges.

\subsubsection{Region $C$}

Third, the bulges in region $\mathrm{C}$ contain a mixture between primary and secondary populations. A careful examination of the spectra of the most extreme bulges in region $\mathrm{C}$ does not cast suspicion on the quality of the measurements. The fraction of barred galaxies in region $\mathrm{C}$ is normal (14 out of 36 objects), at variance with region B: in

\footnotetext{
${ }^{4}$ We note that one of the two barred galaxies (NGC 2339) appears chaotic, and that the other (NGC 3718) is strongly distorted, with a dust lane perpendicular to the bar: they thus appear peculiar.
} 
the latter, the barred fraction is lower (9 out of 34 ), and this figure decreases even more as objects closer to the model of primary population are considered (2 out of 13 ). Unbarred galaxies in region $\mathrm{C}$ could be objects where the bar has already disappeared, or where the accretion comes from outside material.

Lets us now discuss the position of regions $\mathrm{A}, \mathrm{B}$, and $\mathrm{C}$ in the $<\mathrm{Fe}>$ vs. $\mathrm{Mg}_{2}$, and $\sigma_{0}$ vs. $\mathrm{Mg}_{2}$ planes. In Fig. $6 \mathrm{~b}$, the galaxies from these regions are represented by different symbols. The continuous line is the $\mathrm{Mg}_{2}$ vs. $\sigma_{0}$ relation for elliptical galaxies. The solution represented is $\mathrm{Mg}_{2}=0.181 \log \left(\sigma_{0}\right)-0.116$; it corresponds to the solution adopted in Dressler et al. (1987), $\mathrm{Mg}_{2}=$ $0.181 \log \left(\sigma_{0}\right)-0.13$, shifted by $0.014 \mathrm{mag}$ to account for the difference in the effective aperture (see Sect. 4.2). It is in very good agreement with the majority of recent determinations with a similar algorithm (the customary leastsquares fit): $\mathrm{Mg}_{2}=0.199 \log \left(\sigma_{0}\right)-0.168$ in Colless et al. (1999), $\mathrm{Mg}_{2}=0.191 \log \left(\sigma_{0}\right)-0.127$ in K2000. The relation fitted to our data for elliptical galaxies is also similar.

The slope globally observed for the bulges is about twice that observed for elliptical galaxies and only the brightest bulges fall on the line for elliptical galaxies. It is interesting to note that JMA96 find the bulges in fair agreement with elliptical galaxies (they refer to an unpublished source for the $\mathrm{Mg}_{2}$ vs. $\sigma_{0}$ relation for ellipticals, but it is consistent with the well-accepted relation). However, they could only draw the relation for 15 bulges because they were lacking $\sigma_{0}$ measurements for the others, and unfortunately this biases their sample against galaxies with low $\mathrm{Mg}_{2}$ and $\sigma_{0}$, for which internal kinematics are intrinsically difficult to measure. Notwithstanding, a close examination of their Fig. 4 shows a trend, similar to our result, of the smaller bulges to lie below the relation.

If we take the relation for elliptical galaxies as a reference, the bulges, and in particular those exhibiting [OIII] $\lambda 5007 \AA$ emission, have significantly lower $\mathrm{Mg}_{2}$. Hence, the large apparent slope of the $\mathrm{Mg}_{2}$ vs. $\sigma_{0}$ relation is probably the addition of the universal slope due to the mass-metallicity relation with an additional trend due to the correlation of the mass with the mean age of the population.

Furthermore, the galaxies of regions $\mathrm{A}, \mathrm{B}$, and $\mathrm{C}$ also populate different regions in Fig. 6b. The galaxies of region $\mathrm{A}$ are, not unexpectedly, isolated in the lower part of the diagram. Bulges in region B tightly follow the $\mathrm{Mg}_{2}$ vs. $\sigma_{0}$ relation for elliptical galaxies, while those from region $\mathrm{C}$ are intermediate between $\mathrm{A}$ and $\mathrm{B}$. This is noticeable since the distinction between $\mathrm{B}$ and $\mathrm{C}$ does not simply reflect a difference in mass. This separation in Fig. $6 \mathrm{~b}$ gives support for the hypothesis of the contribution of a secondary population in galaxies of region $\mathrm{B}$.

A recent work (Trager et al. 2000b) presents a very tight mass-metallicity relation for a large sample of ellipticals, with only a small deviation for young objects. However, their relation is $\operatorname{Mg} b$ vs. $\sigma_{0}$, and they explain how this index is able to conceal age differences efficiently (however M 32, as a typical example, clearly deviates toward lower indices); the $\mathrm{Mg}_{2}$ relation, although more scattered, is known to present a more pronounced trend for young galaxies, and Fig. 5 of Trager et al. (1998), for example, shows this unambiguously. Our small (and young) bulges, whose $\sigma_{0}$ distribution is much richer in low values $\left(\lesssim 100 \mathrm{~km} \mathrm{~s}^{-1}\right.$ ) than the ellipticals of Trager et al., may therefore be considered as populating an extension of the relation for ellipticals, with a signature of their difference in evolution.

\section{Conclusion}

We have presented the $\mathrm{Mg}_{2}$, Fe 5270 and Fe 5335 indices for 89 bulges of spiral galaxies from the Héraudeau et al. sample, extracted with Hypercat pipeline procedures. The mean error is $0.009 \mathrm{mag}$ on $\mathrm{Mg}_{2}$, and $0.3 \AA$ on Fe 5270 and $\mathrm{Fe} 5335$. These indices are measured according to the Lick definition, and corrected for galaxy internal velocity dispersion. To take into account the radial gradients, we have normalized the measurements to a standard aperture of $0.2 h^{-1} \mathrm{kpc}$, adapted to the present class of objects. This work doubles the amount of data already available.

Our analysis can be summarized as follows:

- The metal indices do correlate with mass indicators. We confirm the well-known relation between $\mathrm{Mg}_{2}$ and $\sigma_{0}$, and we find a tighter relation between $\mathrm{Mg}_{2}$ and $V_{\text {rot }}^{\mathrm{m}}$; more unexpectedly, we find that $<\mathrm{Fe}>$ also correlates with $V_{\text {rot }}^{\mathrm{m}}$, and (marginally) with $\sigma_{0}$. A justification of these relations may be presented as follows: in the range of bright galaxies, the regression lines reflect mass-metallicity relations, gradually replaced, toward fainter objects, by mass-age relations;

- We present a framework for the formation of bulges: a primary population from the initial collapse, mixed in variable proportion with a secondary population which results from secular evolution (either within the disk, e.g., by the evaporation of a bar, or by accretion of extragalactic material). This combination of the two widely discussed formation scenarios fits within the conclusions of Wyse (1999). Although large bulges contain a significantly higher fraction of the primary population, this proportion is not a direct function of the galaxy mass; it is also heavily influenced by morphological characteristics like the presence (and efficiency) of a bar, and the contribution of the disk.

Acknowledgements. We have made use of the LEDA database (http://leda.univ-lyon1.fr). We thank V. Golev for valuable help and discussions. We are indebted to the anonymous referee for his comments. We are grateful to the director and staff of the Observatoire de Haute-Provence for their constant support of our ongoing observational program.

\section{References}

Abraham, R. G., Ellis, R. S., Fabian, A. C., Tanvir, N. R., \& Glazebrook, K. 1999, MNRAS, 303, 641 
Aguerri, J. A. L. 1999, A\&A, 351, 43

Balcells, M., \& Peletier, R. F. 1994, AJ, 107, 135

Beauchamp, D., \& Hardy, E. 1997, AJ, 113, 1666

Bender, R., Burstein, D., \& Faber, S. M. 1992, ApJ, 399, 462

Bender, R., Burstein, D., \& Faber, S. M. 1993, ApJ, 411, 153

Bonatto, C., Pastoriza, M. G., Alloin, D., \& Bica, E. 1998, A\&A, 334, 439

Borges, A. C., Idiart, T. P., de Freitas Pacheco, J. A., \& Thevenin, F. 1995, AJ, 110, 2408

Bouwens, R., Cayón, L., \& Silk, J. 1999, ApJ, 516, 77

Cardiel, N., Gorgas, J., Cenarro, J., \& Gonzáles, J. J. 1998, A\&AS, 127, 597

Carlberg, R. G. 1984, ApJ, 286, 403

Carollo, C. M., Danziger, I. J., \& Buson, L. 1993, MNRAS, 265,553

Colless, M., Burstein, D., Davies, R. L., et al. 1999, MNRAS, 303, 813

Davidge, T. J. 2000, AJ, 119, 748

Davies, R. L., Sadler, E. M., \& Peletier, R. F. 1993, MNRAS, 262,650

De Jong, R. S. 1996, A\&A, 313, 45

Deslisle, S. 1998, Ph.D. Thesis, Laval Univ., Québec

Dressler, A., Lynden-Bell, D., Burstein, D., et al. 1987, ApJ, 313,42

Faber, S. M., Worthey, G., \& Gonzáles, J. J. 1992, in The Stellar Populations of Galaxies, ed. B. Barbuy, \& A. Renzini, Proceedings of IAU Symp. 149 (Kluwer Academic Publishers, Dordrecht), 255

Fich, M., \& Tremaine, S. 1991, ARA\&A, 29, 409

Fisher, D., Franx, M., \& Illingworth, G. 1996, ApJ, 459, 110

Fisher, D. 1997, AJ, 113, 950

Friedli, D., \& Benz, W. 1995, A\&A, 301, 649

Friedli, D., \& Benz, W. 1993, A\&A, 268, 65

Golev, V., Prugniel, Ph., Simien, F., \& Longhetti, M. 1999, A\&AS, 136, 519 (GPSL99)

Golev, V., \& Prugniel, Ph. 1998, A\&AS, 132, 255

González, J. J., Faber, S. M., \& Worthey, G. 1993, A\&AS, 183, 4206

Goudfrooij, P., \& Emsellem, E. 1996, A\&A, 306, L45 (GE96)

Goudfrooij, P., Gorgas, J., \& Jablonka, P. 1999, Ap\&SS, 269270, 109

Graham, A. W., \& Prieto, M. 1999, ApJ, 524, L23

Hasan, H., \& Norman, C. 1990, ApJ, 361, 69

Henry, R. B. C., \& Worthey, G. 1999, PASP, 111, 919

Héraudeau, Ph. 1996, Ph.D. Thesis, Univ. of Lyon

Héraudeau, Ph., \& Simien, F. 1998, A\&AS, 133, 317 (H1)

Héraudeau, Ph., Simien, F., Maubon, G., \& Prugniel, Ph. 1999, A\&AS, 136, 509 (H2)

Idiart, T. P., de Freitas Pacheco, J. A., \& Costa, R. D. D. 1996, AJ, 111, 1169

Idiart, T. P., de Freitas Pacheco, J. A., \& Costa, R. D. D. 1996 AJ, 112, 2541 (IPC96)

Jablonka, P., Martin, P., \& Arimoto, N. 1996, AJ, 112, 1415 (JMA96)

Jacoby, G., Hunter, D., \& Christian, C. 1984, ApJS, 56, 257

Jørgensen, I., Franx, M., \& Kjoergaard, P. 1995, MNRAS, 276 , 1341 (JFK95)

Jørgensen, I. 1997, MNRAS, 288, 161

Jørgensen, I. 1999, MNRAS, 306, 607
Kauffmann, G. 1996, MNRAS, 281, 487

Kent, S. M. 1985, ApJS, 59, 115

Kormendy, J. 1985, ApJ, 292, L9

Kuntschner, H. 2000, MNRAS, 315, 184 (K2000)

Leitherer, C., Alloin, D., Fritze-v. Alvensleben, U., et al. 1996, PASP, 108, 996

Longhetti, M., Rampazzo, R., Bressan, A., \& Chiosi, C. 1998, A\&AS, 130, 251

Martinet, L., \& Friedli, D. 1997, A\&A, 323, 363

Noguchi, M. 1999, ApJ, 514, 77

Norman, C. A., Sellwood, J. A., \& Hasan, H. 1996, ApJ, 462, 114

O'Connell, R. W. 1976, ApJ, 206, 370

Paturel, G., Andernach, H., Bottinelli, L., et al. 1997, A\&AS, 124, 109

Peletier, R. F., Balcells, M., Davies, R. L., et al. 1999, MNRAS, 310,703

Peterson, R. C. 1976, ApJ, 210, L123

Proctor, R. N., Sansom, A. E., \& Reid, N. 2000, MNRAS, 311, $37 \mathrm{P}$

Prugniel, Ph., \& Simien, F. 1994, A\&A, 112, L1

Prugniel, Ph., \& Simien, F. 1996, A\&A, 309, 749

Prugniel, Ph., Golev, V., \& Maubon, G. 1999, A\&A, 346 , L25

Renzini, A. 1999, in The Formation of Galactic Bulges, ed. C. M. Carollo, H. C. Ferguson, \& R. F. G. Wyse (Cambridge Univ. Press), Cambridge Contemporary Astrophysics Series, 9

Rich, R. M. 1999, in The Formation of Galactic Bulges, ed. C. M. Carollo, H. C. Ferguson, \& R. F. G. Wyse (Cambridge Univ. Press), Cambridge Contemporary Astrophysics Series, 54

Sil'Chenko, O. K., Burenkov, A. N., \& Vlasyuk, V. V. 1998, A\&A, 337, 349

Simien, F., \& de Vaucouleurs, G. 1986, ApJ, 302, 564

Simien, F., \& Prugniel, Ph. 1997a, A\&AS, 112, 521

Simien, F., \& Prugniel, Ph. 1997b, A\&AS, 126, 15

Simien, F., Prugniel, Ph. 1997c, A\&AS, 126, 519

Trager, S. C., Worthey, G., Faber, S. M., Burstein, D., \& González, J. J. 1998, ApJS, 116, 1

Trager, S. C., Faber, S. M., Worthey, G., \& González, J. J. 2000, AJ, 119, 1645

Trager, S. C., Faber, S. M., Worthey, G., \& González, J. J. 2000, AJ, 120, 165

Vanzi, L., Alonso-Herrero, A., \& Rieke, G. H. 1998, ApJ, 504, 93

Weiss, A., Peletier, R. F., \& Matteucci, F. 1995, A\&A, 296, 73

Worthey, G., Faber, S. M., González, J. J., \& Burstein, D. 1994, ApJS, 94, 687

Worthey, G., \& Ottaviani, D. L. 1997, ApJS, 111, 377

Worthey, G. 1998, PASP, 110, 888

Wyse, R. F. G., Gilmore, G., \& Franx, M. 1997, ARA\&A, 35, 637

Wyse, R. F. G. 1999, in The Formation of Galactic Bulges, ed. C. M. Carollo, H. C. Ferguson, \& R. F. G. Wyse (Cambridge Univ. Press), Cambridge Contemporary Astrophysics Series, 195

Zaritsky, D., Kennicutt, R. C. Jr., \& Huchra, J. P. 1994, ApJ, 420,87 